\title{
Bounds on size-dependent behaviour of composites
}

\section{S. Saeb, P. Steinmann \& A. Javili}

To cite this article: S. Saeb, P. Steinmann \& A. Javili (2018) Bounds on size-dependent behaviour of composites, Philosophical Magazine, 98:6, 437-463, DOI: 10.1080/14786435.2017.1408967

To link to this article: https://doi.org/10.1080/14786435.2017.1408967

册 Published online: 04 Dec 2017.

Submit your article to this journal ¿

Џ Article views: 144

View Crossmark data \lceil 


\title{
Bounds on size-dependent behaviour of composites
}

\author{
S. Saeb ${ }^{a}$, P. Steinmann ${ }^{a}$ and A. Javili ${ }^{b}$ \\ ${ }^{a}$ Chair of Applied Mechanics, University of Erlangen-Nuremberg, Erlangen, Germany; ${ }^{b}$ Department of \\ Mechanical Engineering, Bilkent University, Ankara, Turkey
}

\begin{abstract}
Computational homogenisation is a powerful strategy to predict the effective behaviour of heterogeneous materials. While computational homogenisation cannot exactly compute the effective parameters, it can provide bounds on the overall material response. Thus, central to computational homogenisation is the existence of bounds. Classical firstorder computational homogenisation cannot capture size effects. Recently, it has been shown that size effects can be retrieved via accounting for elastic coherent interfaces in the microstructure. The primary objective of this contribution is to present a systematic study to attain computational bounds on the sizedependent response of composites. We show rigorously that interface-enhanced computational homogenisation introduces two relative length scales into the problem and investigate the interplay between them. To enforce the equivalence of the virtual power between the scales, a generalised version of the Hill-Mandel condition is employed, and accordingly, suitable boundary conditions are derived. Macroscopic quantities are related to their microscopic counterparts via extended average theorems. Periodic boundary conditions provide an effective behaviour bounded by traction and displacement boundary conditions. Apart from the bounds due to boundary conditions for a given size, the size-dependent response of a composite is bounded, too. The lower bound coincides with that of a composite with no interface. Surprisingly, there also exists an upper bound on the size-dependent response beyond which the expected 'smaller is stronger' trend is no longer observed. Finally, we show an excellent agreement between our numerical results and the corresponding analytical solution for linear isotropic materials which highlights the accuracy and broad applicability of the presented scheme.
\end{abstract}

\section{ARTICLE HISTORY}

Received 3 August 2017 Accepted 21 November 2017

\section{KEYWORDS}

Computational homogenisation; finite deformation; interface elasticity; size effects

\section{Introduction}

Almost all materials ranging from biological matter to industrial components consist of multiple constituents and possess heterogeneous structure at a certain scale of observation. Predicting the behaviour of such materials is a serious challenge as their overall response originates from their underlying

CONTACT A. Javili $\otimes$ ajavili@bilkent.edu.tr 
microstructures. The microstructures generally differ from one material to another in material properties, volume fraction, shape and orientation of the constituents. In addition to these physical and geometrical effects, it is wellknown that the interfaces between the constituents exhibit properties different from those associated with the bulk material and can lead to major alternation of the material response. Interface effects are particularly important for multiphase nano-materials where the area-to-volume ratio is significantly large. Obviously, motivated by their ever-increasing applications due to supreme mechanical properties, higher thermal stability and better durability, understanding the behaviour of such materials is of great importance.

Investigating the behaviour of nano-structured materials incorporating interface effects has been the subject of numerous studies [1-15]. The term 'interface' usually refers to a zero-thickness model that represents the finite thickness 'interphase' between different phases of the microstructure. Interface effects can be modelled using several continuum approaches which are thoroughly reviewed in [16,17]. In this contribution, we use the interface elasticity theory [18-22] which is originally based on the classical surface elasticity theory of Gurtin [23] that accounts for surfaces with their own constitutive behaviour [24]. The interface elasticity model does not allow for a displacement jump across the interface; however, the traction jump due to the stress along the interface may exist and follows the generalised Young-Laplace equation [25]. For generalised interfaces, see [26-28], among others. Extension of interface models to account for curvature dependence has been studied in [29-32].

In order to predict the response of a heterogeneous material, several multiscale techniques have been developed in the past. Reviews of the different multiscale approaches can be found in [33-36]. Among these techniques, first-order computational homogenisation method or more specifically direct micro-tomacro transition method has become the most popular technique. An extensive body of literature is devoted to study this technique, among which we refer to [37-52]. The main assumption of homogenisation is that the microstructure of the heterogeneous material is far smaller than the characteristic length of the macrostructure. This separation of scales allows to view the problem as two coupled subproblems at the macro- and micro-scale. It is assumed that the constitutive response of the microstructure is known and, in an average sense, results in the effective response of the macrostructure. Usually, both the macro-problem and the micro-problem are discretised and solved using the finite element method [53-56]. The statistically similar sample of the microstructure is commonly referred to as representative volume element (RVE). For further details and the identification of RVEs, see [57-59]. The major limitation associated with classical first-order computational homogenisation is that it lacks a physical length scale and, thus, fails to account for the size-dependent behaviour of the material response commonly referred to as size effect. It has been recently shown that including interfaces at the micro-scale introduces a physically interpretable 
length scale [8,60] and agrees well with atomistic simulations [61], see also [62,63]. Another methodology to introduce a physical length scale at the micro-scale is to employ second-order computational homogenisation developed in [64] where higher order gradients are incorporated in the material response.

Central to computational homogenisation is the Hill-Mandel condition [65] that enforces the equivalence of the virtual power between the micro- and the macro-scale. The classical Hill-Mandel condition does not account for interfaces. Here, we employ an extended form of the Hill-Mandel condition proposed in [66] for an interface-enhanced computational homogenisation framework. In order to satisfy the Hill-Mandel condition, appropriate boundary conditions have to be imposed on the micro-scale problem. Among various options satisfying the Hill-Mandel condition, we report on displacement (DBC), periodic (PBC) and traction (TBC) boundary conditions. In classical first-order computational homogenisation in elasticity, it is accepted that the results associated with $\mathrm{DBC}$ and TBC overestimate and underestimate the results of the PBC, respectively [67-69], see also [70] for second-order computational homogenisation. The results obtained from different boundary conditions tend to converge to an effective value when the size of the microstructure and the number of its heterogeneities increases [71,72].

The influence of elastic surfaces using $\mathrm{PBC}$ on the response of the material in the context of the computational homogenisation and large deformations has been recently studied $[73,74]$. Utilising the periodic boundary conditions is commonly justified by the fact that they often produce the most intermediate results as compared to other boundary conditions. However, it has been shown that the effective response obtained under $\mathrm{PBC}$ might underestimate $\mathrm{TBC}$ or overestimate DBC [75]. In this contribution, we detail on the influence of different boundary conditions on the overall response of the material when elastic interfaces are included. We investigate the effective response of various microstructures which are different in size, number of inclusions and material properties. In addition, we compare our numerical results with the analytical solution of several microproblems in which the effects of interfaces are considered.

The remainder of this manuscript is organised as follows. The governing equations of the macro- and micro-problem, Hill-Mandel condition and the transition between the scales are discussed in Section 2. Section 3 details on the finite element formulation of the micro-problem. The applicability of the presented framework and also the influence of different boundary conditions on the macroscopic response are demonstrated through several numerical examples in Section 4. Section 5 provides a systematic comparison between the numerical results and the corresponding analytical solution. Finally, Section 6 concludes this work and provides further outlook. 


\section{Theory}

This section details on theoretical aspects of modelling large deformations of a heterogeneous material accounting for elastic interfaces between the constituents. Based on the assumption of separation of scales, the problem is separated into two coupled problems at the micro- and macro-scale. The constitutive response of the microstructure is assumed to be known and results in the overall behaviour of the macrostructure through homogenisation. This work is based on the first-order strain-driven computational homogenisation. That is, the macro deformation gradient is the input of the micro-problem and the macroscopic Piola stress is calculated via a suitable averaging process.

Consider a heterogeneous material at the macro-scale that takes the material configuration ${ }^{\mathrm{M}} \mathcal{B}_{0}$ at time $t=0$ and the spatial configuration ${ }^{\mathrm{M}} \mathcal{B}_{t}$ at any time $t>0$, as shown in Figure 1. The boundary of the material and spatial configurations are denoted ${ }^{\mathrm{M}} \mathcal{S}_{0}$ and ${ }^{\mathrm{M}} \mathcal{S}_{t}$ with the outward unit normal vector ${ }^{\mathrm{M}} \boldsymbol{N}$ and ${ }^{\mathrm{M}} \boldsymbol{n}$, respectively. The position vector of a point in ${ }^{\mathrm{M}} \mathcal{B}_{0}$ is denoted ${ }^{\mathrm{M}} \boldsymbol{X}$ which is mapped to its spatial counterpart ${ }^{\mathrm{M}} \boldsymbol{x}$ via the nonlinear deformation map ${ }^{\mathrm{M}} \boldsymbol{x}=$ ${ }^{\mathrm{M}} \boldsymbol{\varphi}\left({ }^{\mathrm{M}} \boldsymbol{X}\right)$. A material line element $\mathrm{d}^{\mathrm{M}} \boldsymbol{X}$ is mapped to its spatial counterpart $\mathrm{d}^{\mathrm{M}} \boldsymbol{x}$ with the macroscopic linear deformation map as $\mathrm{d}^{\mathrm{M}} \boldsymbol{x}={ }^{\mathrm{M}} \boldsymbol{F} \cdot \mathrm{d}^{\mathrm{M}} \boldsymbol{X}$ with ${ }^{\mathrm{M}} \boldsymbol{F}$ being the macroscopic deformation gradient defined as ${ }^{\mathrm{M}} \boldsymbol{F}={ }^{\mathrm{M}} G r a{ }^{\mathrm{M}} \boldsymbol{\varphi}$.

The governing equations of the macro-problem are the balance of linear and angular momentum. Assuming a quasi-static problem and excluding body forces, the balance of linear momentum takes the form

$$
{ }^{\mathrm{M}}{ }^{\mathrm{Div}}{ }^{\mathrm{M}} \boldsymbol{P}=\mathbf{0} \quad \text { in }{ }^{\mathrm{M}} \mathcal{B}_{0} \quad \text { subject to } \quad{ }^{\mathrm{M}} \boldsymbol{P} \cdot{ }^{\mathrm{M}} \boldsymbol{N}={ }^{\mathrm{M}} \boldsymbol{t}_{0} \quad \text { on }{ }^{\mathrm{M}} \mathcal{S}_{0},
$$

in which ${ }^{\mathrm{M}} \boldsymbol{P}$ is the macroscopic Piola stress and ${ }^{\mathrm{M}} \boldsymbol{t}_{0}$ denotes the traction on the boundary ${ }^{\mathrm{M}} \mathcal{S}_{0}$. Balance of angular momentum at the macro-scale is equivalent to the symmetry of the Cauchy stress in the bulk or equivalently ${ }^{\mathrm{M}} \boldsymbol{P} \cdot{ }^{\mathrm{M}} \boldsymbol{F}^{\mathrm{t}}=$ ${ }^{\mathrm{M}} \boldsymbol{F} \cdot{ }^{\mathrm{M}} \boldsymbol{P}^{\mathrm{t}}$.

The interface elasticity enters the macro-problem implicitly, and it is only included explicitly at the micro-scale. Therefore, defining the kinematics of the problem at the micro-scale requires including the details related to the elastic interfaces too. Let $\mathcal{B}_{0}$ define the RVE in the material configuration which consists of two disjoint subdomains, $\mathcal{B}_{0}^{+}$and $\mathcal{B}_{0}^{-}$, bonded together by the interface $\mathcal{I}_{0}$. The spatial configuration is defined analogously. The boundaries of $\mathcal{B}_{0}^{+}$and $\mathcal{B}_{0}^{-}$ are denoted $\partial \mathcal{B}_{0}^{+}$and $\partial \mathcal{B}_{0}^{-}$, respectively. The external boundary of $\mathcal{B}_{0}$ is denoted $\mathcal{S}_{0}$ and does not include the interface $\mathcal{I}_{0}$, thus $\mathcal{S}_{0}=\left[\partial \mathcal{B}_{0}^{+} \cup \partial \mathcal{B}_{0}^{-}\right] \backslash \mathcal{I}_{0}$. The boundary of the interface $\mathcal{I}_{0}$ is denoted $\mathcal{L}_{0}$. The outward unit normal to $\mathcal{S}_{0}$ is denoted $N$ while the outward unit normal to $\mathcal{L}_{0}$ is $\widetilde{N}$ and is tangent to $\mathcal{I}_{0}$, not necessarily aligned with $N$. The normal to the interface $\mathcal{I}_{0}$ is denoted $\bar{N}$. Let $\boldsymbol{X}$ define the position vector of a point in $\mathcal{B}_{0}$ mapped to its spatial counterpart $\boldsymbol{x}$ via $x=\varphi(X)$. In a similar fashion, we define the position vector of a point on the interface $\mathcal{I}_{0}$ as $\bar{X}$ mapped to its spatial counterpart $\bar{x}$ through $\bar{x}=\bar{\varphi}(\bar{X})$. 
The bulk and the interface deformation gradients are defined as $\boldsymbol{F}=\operatorname{Grad} \boldsymbol{\varphi}$ and $\overline{\boldsymbol{F}}=\overline{\operatorname{Grad}} \overline{\boldsymbol{\varphi}}=\boldsymbol{F} \cdot \overline{\boldsymbol{I}}$, respectively, in which the interface identity tensor is $\bar{I}=I-\bar{N} \otimes \bar{N}$. The governing equations of the problem at the micro-scale are the balances of linear and angular momentum. The balance of linear momentum in the bulk and on the interface reads

$$
\begin{array}{lcccc}
\operatorname{Div} \boldsymbol{P}=\mathbf{0} & \text { in } \mathcal{B}_{0} & \text { subject to } & \boldsymbol{P} \cdot \boldsymbol{N}=\boldsymbol{t}_{0} & \text { on } \mathcal{S}_{0}, \\
\overline{\mathrm{Div}} \overline{\boldsymbol{P}}+\llbracket \boldsymbol{P} \rrbracket \cdot \overline{\boldsymbol{N}}=\mathbf{0} & \text { on } \mathcal{I}_{0} & \text { subject to } & \overline{\boldsymbol{P}} \cdot \widetilde{\boldsymbol{N}}=\overline{\boldsymbol{t}}_{0} & \text { on } \mathcal{L}_{0},
\end{array}
$$

respectively, where $\overline{\operatorname{Div}}\{\bar{\bullet}\}=\overline{\operatorname{Grad}}\{\bar{\bullet}\}: \overline{\boldsymbol{I}}$. The stresses in the bulk and on the interface are denoted by $\boldsymbol{P}$ and $\overline{\boldsymbol{P}}$, respectively. Moreover, $\llbracket \boldsymbol{P} \rrbracket \cdot \overline{\boldsymbol{N}}$ represents the jump of the traction across the interface and is equal to $\llbracket \boldsymbol{P} \rrbracket \cdot \overline{\boldsymbol{N}}=\left[\boldsymbol{P}^{+}-\boldsymbol{P}^{-}\right] \cdot \overline{\boldsymbol{N}}$, where $\boldsymbol{P}^{+}$and $\boldsymbol{P}^{-}$represent the Piola stresses in $\mathcal{B}_{0}^{+}$and $\mathcal{B}_{0}^{-}$, respectively. The interface Piola stress is a second-order superficial tensor possessing the property $\overline{\boldsymbol{P}} \cdot \overline{\boldsymbol{N}}=\mathbf{0}$. Note when elastic interfaces are considered, $\overline{\mathrm{Div}} \overline{\boldsymbol{P}}$ does not vanish and the traditional traction continuity condition across the interface no longer holds. The local form of the balance of angular momentum is equivalent to the symmetry of the Cauchy stress in the bulk and on the interface. Alternatively, balance of angular momentum in the bulk and on the interface reads $\boldsymbol{P} \cdot \boldsymbol{F}^{\mathrm{t}}=$ $\boldsymbol{F} \cdot \boldsymbol{P}^{\mathrm{t}}$ and $\overline{\boldsymbol{P}} \cdot \overline{\boldsymbol{F}}^{\mathrm{t}}=\overline{\boldsymbol{F}} \cdot \overline{\boldsymbol{P}}^{\mathrm{t}}$, respectively.

Next, an appropriate micro-to-macro transition technique should be established to relate the macroscopic quantities to their microscopic counterparts. In classical computational homogenisation, macroscopic quantities are commonly related to their microscopic counterparts through volume averaging over the microstructure. Nevertheless, the classical definitions do not hold when interfaces are present. Following the extended average deformation gradient and stress theorems given in $[60,74]$, the macroscopic deformation gradient and the macroscopic Piola stress for interface-enhanced computational homogenisation are defined as

$$
\begin{aligned}
{ }^{\mathrm{M}} \boldsymbol{F} & =\frac{1}{\mathscr{V}_{0}} \int_{\mathcal{B}_{0}} \boldsymbol{F} \mathrm{d} V=\frac{1}{\mathscr{V}_{0}} \int_{\mathcal{S}_{0}} \boldsymbol{\varphi} \otimes \boldsymbol{N} \mathrm{d} A, \\
{ }^{\mathrm{M}} \boldsymbol{P} & =\frac{1}{\mathscr{V}_{0}} \int_{\mathcal{B}_{0}} \boldsymbol{P} \mathrm{d} V+\frac{1}{\mathscr{V}_{0}} \int_{\mathcal{I}_{0}} \overline{\boldsymbol{P}} \mathrm{d} A=\frac{1}{\mathscr{V}_{0}} \int_{\mathcal{S}_{0}} \boldsymbol{t}_{0} \otimes \boldsymbol{X} \mathrm{d} A+\frac{1}{\mathscr{V}_{0}} \int_{\mathcal{L}_{0}} \overline{\boldsymbol{t}}_{0} \otimes \overline{\boldsymbol{X}} \mathrm{d} L,
\end{aligned}
$$

in which $\mathscr{V}_{0}$ is the total volume surrounded by the external boundary of the RVE. Clearly, the macroscopic deformation gradient definition assumes the same format whether or not the elastic interfaces are taken into account while the definition of the macroscopic Piola stress differs from its definition in classical computational homogenisation. Obviously, in the absence of interface effects, the classical definition of macroscopic Piola stress is recovered from Equation $(4)_{2}$. 

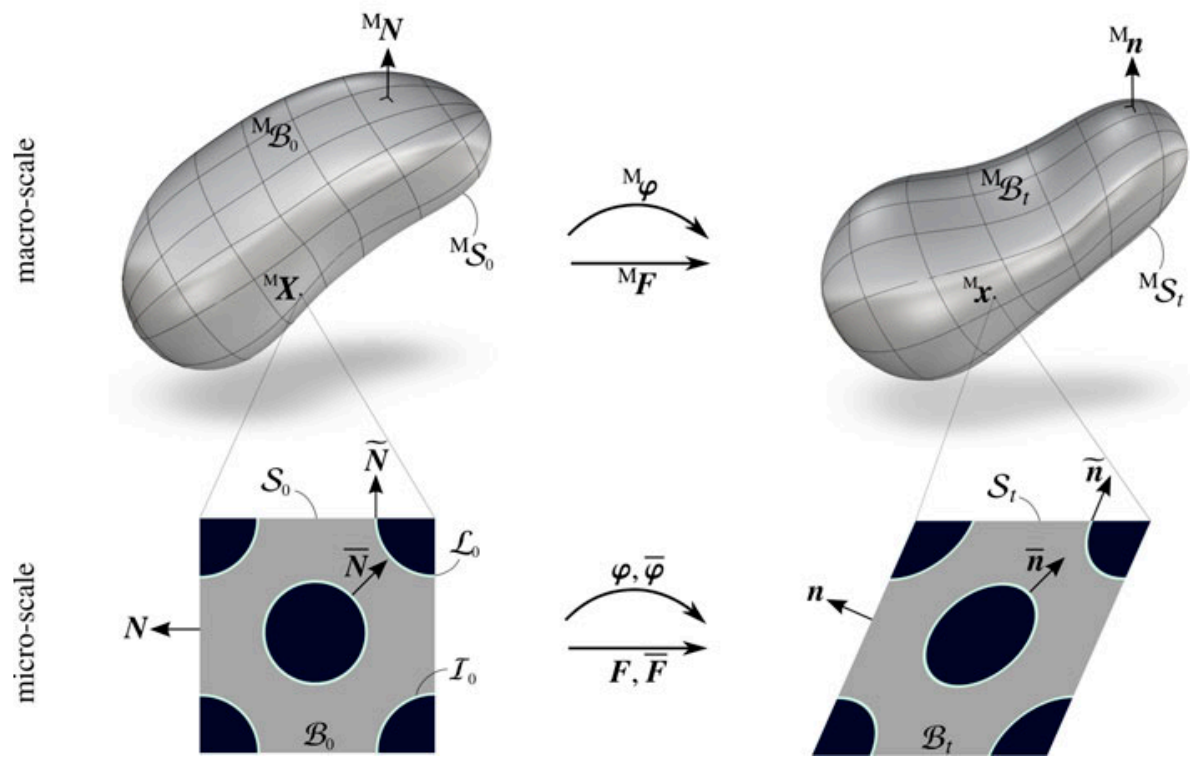

Figure 1. (colour online) Graphical summary of computational homogenisation with elastic interfaces at the micro-level. The material configuration $\mathcal{B}_{0}$ corresponds to a RVE which includes the geometrical information of the macroscopic point ${ }^{{ }^{M}} \boldsymbol{X}$ and is mapped to its spatial configuration through the nonlinear deformation maps $\varphi$ and $\bar{\varphi}$. The local macroscopic response is obtained through solving the associated boundary value problem at the micro-scale.

Finally, in order to derive suitable boundary conditions on the RVE, the virtual power equivalence between the two scales is fulfilled. To do so, we employ the generalised Hill-Mandel condition

$$
{ }^{\mathrm{M}} \boldsymbol{P}: \delta{ }^{\mathrm{M}} \boldsymbol{F}=\frac{1}{\mathscr{V}_{0}} \int_{\mathcal{S}_{0}} \boldsymbol{t}_{0} \cdot \delta \boldsymbol{\varphi} \mathrm{d} A+\frac{1}{\mathscr{V}_{0}} \int_{\mathcal{L}_{0}} \overline{\boldsymbol{t}}_{0} \cdot \delta \overline{\boldsymbol{\varphi}} \mathrm{d} L
$$

Using the Lemma

$$
\frac{1}{\mathscr{V}_{0}} \int_{\mathcal{S}_{0}} \boldsymbol{t}_{0} \cdot \delta \boldsymbol{\varphi} \mathrm{d} A+\frac{1}{\mathscr{V}_{0}} \int_{\mathcal{L}_{0}} \overline{\boldsymbol{t}}_{0} \cdot \delta \overline{\boldsymbol{\varphi}} \mathrm{d} L=\frac{1}{\mathscr{V}_{0}} \int_{\mathcal{B}_{0}} \boldsymbol{P}: \delta \boldsymbol{F} \mathrm{d} V+\frac{1}{\mathscr{V}_{0}} \int_{\mathcal{I}_{0}} \overline{\boldsymbol{P}}: \delta \overline{\boldsymbol{F}} \mathrm{d} A
$$

proven in Appendix 1, the Hill-Mandel condition (5) can alternatively be expressed as

$$
\frac{1}{\mathscr{V}_{0}} \int_{\mathcal{B}_{0}} \boldsymbol{P}: \delta \boldsymbol{F} \mathrm{d} V+\frac{1}{\mathscr{V}_{0}} \int_{\mathcal{I}_{0}} \overline{\boldsymbol{P}}: \delta \overline{\boldsymbol{F}} \mathrm{d} A-{ }^{\mathrm{M}} \boldsymbol{P}: \delta^{\mathrm{M}} \boldsymbol{F}=0
$$


With the aid of the generalised Hill's Lemma proven in Appendix 2 as

$$
\begin{aligned}
& \frac{1}{\mathscr{V}_{0}} \int_{\mathcal{B}_{0}} \boldsymbol{P}: \delta \boldsymbol{F} \mathrm{d} V+\frac{1}{\mathscr{V}_{0}} \int_{\mathcal{I}_{0}} \overline{\boldsymbol{P}}: \delta \overline{\boldsymbol{F}} \mathrm{d} A-{ }^{\mathrm{M}} \boldsymbol{P}: \delta^{\mathrm{M}} \boldsymbol{F} \\
& \quad=\frac{1}{\mathscr{V}_{0}} \int_{\mathcal{S}_{0}}\left[\delta \boldsymbol{\varphi}-\delta^{\mathrm{M}} \boldsymbol{F} \cdot \boldsymbol{X}\right] \cdot \boldsymbol{t}_{0} \mathrm{~d} A+\frac{1}{\mathscr{V}_{0}} \int_{\mathcal{L}_{0}}\left[\delta \overline{\boldsymbol{\varphi}}-\delta^{\mathrm{M}} \boldsymbol{F} \cdot \overline{\boldsymbol{X}}\right] \cdot \overline{\boldsymbol{t}}_{0} \mathrm{~d} L,
\end{aligned}
$$

the Hill-Mandel condition (7) can be rewritten in terms of boundary integrals as

$$
\frac{1}{\mathscr{V}_{0}} \int_{\mathcal{S}_{0}}\left[\delta \boldsymbol{\varphi}-\delta^{\mathrm{M}} \boldsymbol{F} \cdot \boldsymbol{X}\right] \cdot \boldsymbol{t}_{0} \mathrm{~d} A+\frac{1}{\mathscr{V}_{0}} \int_{\mathcal{L}_{0}}\left[\delta \overline{\boldsymbol{\varphi}}-\delta^{\mathrm{M}} \boldsymbol{F} \cdot \overline{\boldsymbol{X}}\right] \cdot \overline{\boldsymbol{t}}_{0} \mathrm{~d} L=0 .
$$

In the remainder of the manuscript, we assume that $\mathcal{L}_{0}=\varnothing$ (i.e. no interface penetrates the boundary of the microstructure) and Equation (5) reduces to

$$
\frac{1}{\mathscr{V}_{0}} \int_{\mathcal{S}_{0}}\left[\delta \boldsymbol{\varphi}-\delta^{\mathrm{M}} \boldsymbol{F} \cdot \boldsymbol{X}\right] \cdot \boldsymbol{t}_{0} \mathrm{~d} A=0
$$

which is the Hill-Mandel condition (in terms of a boundary integral) in its classical format. In view of the Hill-Mandel condition in its various forms (5), (7) and (9), it must be emphasised that the equality sign shall be understood as a conditional equality but not an identity. More precisely, the Hill-Mandel condition is satisfied if and only if the equality holds. In order to satisfy the Hill-Mandel condition, the right-hand side of the identity (10) should vanish identically which can be sufficiently guaranteed through the following canonical boundary conditions:

- Displacement boundary conditions (DBC)

$$
\rightsquigarrow \varphi={ }^{\mathrm{M}} \boldsymbol{F} \cdot \boldsymbol{X} \quad \text { on } \mathcal{S}_{0} \text {, }
$$

- Periodic boundary conditions (PBC)

$\rightsquigarrow\left[\varphi-{ }^{\mathrm{M}} \boldsymbol{F} \cdot \boldsymbol{X}\right]$ : periodic and $\boldsymbol{t}_{0}$ : anti-periodic on $\mathcal{S}_{0}$,

- Traction boundary conditions (TBC)

$$
\rightsquigarrow \quad t_{0}={ }^{\mathrm{M}} \boldsymbol{P} \cdot \boldsymbol{N} \quad \text { on } \mathcal{S}_{0} \text {. }
$$

It can be readily shown that the aforementioned boundary conditions fulfil the balance of angular momentum at the macro-scale. This fact has been proven in $[35,76]$ for classical computational homogenisation. Extension of the proofs for interface-enhanced computational homogenisation follows the same steps and is not presented here for the sake of space.

Remark on the relative length scales in the micro-problem. In the context of interface elasticity, the interface is assumed to be a zero-thickness model and, thus, has a lower dimension compared to the bulk. The macroscopic Piola stress $(4)_{2}$ can be written as

$$
{ }^{\mathrm{M}} \boldsymbol{P}=\frac{1}{\mathscr{V}_{0}} \int_{\mathcal{B}_{0}} \boldsymbol{P} \mathrm{d} V+\frac{\mathscr{S}_{0}}{\mathscr{V}_{0}} \frac{1}{\mathscr{S}_{0}} \int_{\mathcal{I}_{0}} \overline{\boldsymbol{P}} \mathrm{d} A,
$$



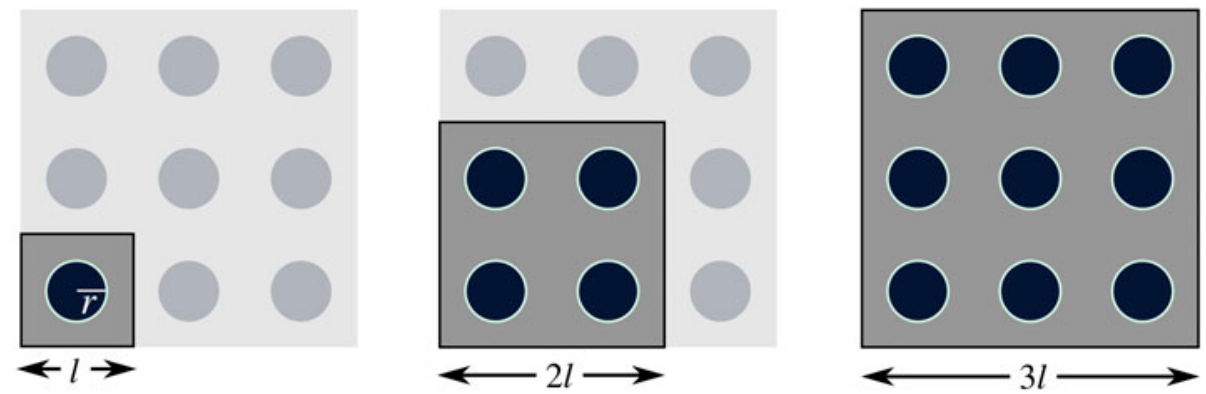

Figure 2. (colour online) The depicted periodic microstructures share the same area-to-volume ratio and yield the same macroscopic response under PBC.

in which $\mathscr{S}_{0}$ refers to the area of the elastic interfaces. Clearly, area-to-volume $\mathscr{S}_{0} / \mathscr{V}_{0}$ has the dimension $[1 / \mathrm{m}]$ and introduces a relative length scale to the micro-problem solution. The greater the area-to-volume ratio, the greater the contribution from the interfaces on the overall response of the microstructure. The second relative length scale introduced in this framework is surface-tobulk energy density $\bar{\psi} / \psi$ which has the dimension $[\mathrm{m}]$. Clearly, the larger the interface material parameters, the larger the influence of the interface on the overall response of the microstructure. Thus, the contribution from the interface on the overall response of the microstructure can be varied by altering the size of the microstructure or changing the interface material parameters. Reducing the size of the microstructure is equivalent to increasing the interface material parameters and leads to more pronounced interface effect on the overall response. Also, it is clear that these two relative length scales might act against each other. That is, for instance, decreasing the size of the microstructure can be counteract by decreasing the interface material properties and vice versa. Note that for a periodic microstructure depicted in Figure 2, $\mathscr{S}_{0} / \mathscr{V}_{0}$ remains constant and equal to $2 \pi r / l^{2}$. Under periodic conditions, the volume average of bulk stresses and surface average of interface stresses are also identical for all the microstructures. Therefore, for constant interface material parameters, the microstructures shown in Figure 2 are all equivalent to each other and yield similar macroscopic behaviour.

\section{Computational aspects}

This section presents the details of the finite element formulation of a boundary value problem at the micro-scale accounting for elastic interfaces. First, the weak forms of the balances of linear momentum (2) and (3) are derived. Second, the weak forms are discretised in space. Finally, the resulting nonlinear system of equations is linearised and solved using the Newton-Raphson scheme.

In order to obtain the weak form of the balance of linear momentum, the lefthand side of Equations (2) and (3) are tested with vector valued test functions, $\delta \varphi$ and $\delta \bar{\varphi}$, respectively, and are integrated over the bulk and interface domain 
in the material configuration. Through combining the resulting equations and employing the bulk and the interface divergence theorems along with considering the superficiality of the interface Piola stress, we obtain the weak form of the balance of linear momentum as

$$
\begin{gathered}
\int_{\mathcal{B}_{0}} \boldsymbol{P}: \operatorname{Grad} \delta \boldsymbol{\varphi} \mathrm{d} V-\int_{\mathcal{S}_{0, \mathrm{~N}}} \delta \boldsymbol{\varphi} \cdot \boldsymbol{t}_{0} \mathrm{~d} A+\int_{\mathcal{I}_{0}} \overline{\boldsymbol{P}}: \overline{\operatorname{Grad}} \delta \overline{\boldsymbol{\varphi}} \mathrm{d} A=0 \\
\forall \delta \boldsymbol{\varphi} \in \mathcal{H}_{0}^{1}\left(\mathcal{B}_{0}\right), \forall \delta \overline{\boldsymbol{\varphi}} \in \mathcal{H}_{0}^{1}\left(\mathcal{I}_{0}\right),
\end{gathered}
$$

where $\mathcal{S}_{0, \mathrm{~N}}$ refers to the Neumann portion of the bulk boundary. Next, the material domain is discretised into sets of bulk, surface and interface finite elements as

$$
\underset{\beta=1}{\mathrm{Abe}} \int_{\mathcal{B}_{0}^{\beta}} \boldsymbol{P}: G r a d \delta \boldsymbol{d} V-\underset{\gamma=1}{\mathrm{Ase}} \int_{\mathcal{S}_{0, \mathrm{~N}}^{\gamma}} \delta \boldsymbol{\varphi} \cdot \boldsymbol{t}_{0} \mathrm{~d} A+\underset{\alpha=1}{\mathrm{~A}} \int_{\mathcal{I}_{0}^{\alpha}} \overline{\boldsymbol{P}}: \overline{G r a d} \delta \overline{\boldsymbol{\varphi}} \mathrm{d} A=0 \text {, }
$$

where \#be, \#se and \#ie represent the number of bulk, surface and interface elements, respectively. The domain of the bulk element $\beta$ is denoted $\mathcal{B}_{0}^{\beta}$. The domain of the surface element $\gamma$ upon which traction is prescribed is denoted $\mathcal{S}_{0, \mathrm{~N}}^{\gamma}$. The interface element $\alpha$ is denoted $\mathcal{I}_{0}^{\alpha}$. The fully discrete weak form of the balance of linear momentum is obtained by replacing the test functions in Equation (13) with their spatial Bubnov-Galerkin approximations and utilising the isoparametric concept as

$$
\boldsymbol{R}^{I}:=\underset{\beta=1}{\# \text { \# }} \int_{\mathcal{B}_{0}^{\beta}} \boldsymbol{P} \cdot G r a d N^{i} \mathrm{~d} V-\underset{\gamma=1}{\# \text { se }} \int_{\mathcal{S}_{0, \mathrm{~N}}^{\gamma}} \boldsymbol{t}_{0} \cdot N^{i} \mathrm{~d} A+\underset{\alpha=1}{\# \text { ie }} \int_{\mathcal{I}_{0}^{\alpha}} \overline{\boldsymbol{P}} \cdot \overline{G r a d} \bar{N}^{i} \mathrm{~d} A=\mathbf{0} .
$$

The fully discrete form of the residual associated with the global node $I$ is denoted by the vector $\boldsymbol{R}^{I}$. In Equation (14), $i$ represents the local node of a finite element corresponding to the global node $I$. The shape functions in the bulk and on the interface associated with the local node $i$ are denoted $N^{i}$ and $\bar{N}^{i}$. The nodal residuals are then collected into a global residual vector $\mathbf{R}$ as

$$
\mathbf{R}=\mathbf{R}(\mathbf{d})=\mathbf{0} \quad \text { with } \quad \mathbf{R}=\left[\boldsymbol{R}^{1} \ldots \boldsymbol{R}^{I} \ldots \boldsymbol{R}^{\# \mathrm{n}}\right]^{\mathrm{t}}
$$

in which $\mathbf{d}$ denotes the unknown global vector of displacements and \#n denotes the number of nodes. Finally, the Newton-Raphson method is utilised to linearise and solve the nonlinear system (15) which yields

$$
\mathbf{R}\left(\mathbf{d}_{n+1}\right)=\mathbf{R}\left(\mathbf{d}_{n}\right)+\left.\frac{\partial \mathbf{R}}{\partial \mathbf{d}}\right|_{n} \cdot \Delta \mathbf{d}_{n}=\mathbf{0} \Rightarrow \Delta \mathbf{d}_{n}=-\mathbf{K}^{-1} \cdot \mathbf{R}\left(\mathbf{d}_{n}\right),
$$

and

$$
\mathbf{d}_{n+1}=\mathbf{d}_{n}+\Delta \mathbf{d}_{n}
$$


where $\mathbf{K}$ is referred to as the global stiffness matrix which is the collection of the assembled nodal stiffness matrices

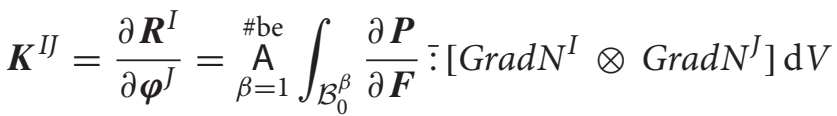

$$
\begin{aligned}
& +\underset{\alpha=1}{\#} \int_{\mathcal{I}_{0}^{\alpha}} \frac{\partial \overline{\boldsymbol{P}}}{\partial \overline{\boldsymbol{F}}} \overline{:}\left[\overline{\mathrm{Grad}} \bar{N}^{I} \otimes \overline{\text { Grad }} \bar{N}^{J}\right] \mathrm{d} A \text {. }
\end{aligned}
$$

The non-standard (double) contraction $\bar{\vdots}$ of a fourth-order tensor $\mathbb{A}$ and a second-order tensor $\boldsymbol{B}$ is a second-order tensor $\boldsymbol{C}=\mathbb{A}: \boldsymbol{B}$ with components $C_{i k}=A_{i j k l} B_{j l}$. The computational implementation of the micro-problem is established upon implementing a specific type of boundary conditions to be imposed on the system of equations (15). Computational aspects of implementing DBC and $\mathrm{PBC}$ in the context of the finite element method and large deformations are straightforward and well established [35]. For implementing TBC, we utilise the semi-Dirichlet boundary conditions proposed recently [77]. The methodology remains almost identical for interface-enhanced computational homogenisation with the difference that the macroscopic Piola stress should be evaluated using the relation $(4)_{2}$ rather than its classical definition.

\section{Numerical examples}

The goal of this section is to illustrate the performance of the presented framework through providing several numerical examples and to study the influence of elastic interfaces on the response of the material under different conditions. In particular, we devise the examples such that the computational bounds on the overall response of the material are clearly demonstrated. We mainly focus on the influence of the inclusion material properties, microstructure size and the choice of the boundary conditions on the macroscopic response. The effective property of interest in this contribution is the $x x$-component of the macroscopic Piola stress. However, other alternatives and valid choices lead to the same trends and conclusions qualitatively. All the following numerical examples are solved using our in-house finite element code.

For the material response of the bulk at the micro-scale, we assume a hyperelastic neo-Hookean energy density per unit volume in the material configuration

$$
\psi(\boldsymbol{F})=\frac{1}{2} \mu[\boldsymbol{F}: \boldsymbol{F}-3-2 \log J]+\frac{1}{2} \lambda \log ^{2} J \quad \text { with } \quad J=\operatorname{det} \boldsymbol{F},
$$

in which $\mu$ and $\lambda$ denote the bulk Lamé parameters. Similarly, for the material response of the interface, we assume a hyperelastic neo-Hookean energy density per unit area in the material configuration

$$
\bar{\psi}(\overline{\boldsymbol{F}})=\frac{1}{2} \bar{\mu}[\overline{\boldsymbol{F}}: \overline{\boldsymbol{F}}-2-2 \log \bar{J}]+\frac{1}{2} \bar{\lambda} \log ^{2} \bar{J} \quad \text { with } \quad \bar{J}=\overline{\operatorname{det}} \overline{\boldsymbol{F}},
$$


where $\bar{\mu}$ and $\bar{\lambda}$ are the interface material parameters. For the definition of the interface determinant, see [78]. In a two-dimensional structure, the interface is a one-dimensional manifold which resists against its length change. Thus, it is sufficient to introduce only one material parameter to define the behaviour of the interfaces as

$$
\bar{\psi}(\overline{\boldsymbol{F}})=\frac{1}{2} \bar{\mu}[\overline{\boldsymbol{F}}: \overline{\boldsymbol{F}}-2-2 \log \bar{J}]
$$

The associated bulk and interface Piola stress tensors are derived as

$$
\begin{gathered}
\boldsymbol{P}:=\frac{\partial \psi}{\partial \boldsymbol{F}}=\mu\left[\boldsymbol{F}-\boldsymbol{F}^{-\mathrm{t}}\right]+\lambda \log J \boldsymbol{F}^{-\mathrm{t}}, \\
\overline{\boldsymbol{P}}:=\frac{\partial \bar{\psi}}{\partial \overline{\boldsymbol{F}}}=\bar{\mu}\left[\overline{\boldsymbol{F}}-\overline{\boldsymbol{F}}^{-\mathrm{t}}\right]+\bar{\lambda} \log \bar{J} \overline{\boldsymbol{F}}^{-\mathrm{t}},
\end{gathered}
$$

In order to solve the system of Equation (15) using the Newton-Raphson method, in addition to the Piola stresses, the bulk Piola tangent $\mathbb{A}$ and the interface Piola tangent $\overline{\mathbb{A}}$ are calculated as

$$
\begin{aligned}
\mathbb{A}:= & \frac{\partial^{2} \psi}{\partial \boldsymbol{F}^{2}}=\mu\left[\boldsymbol{I} \bar{\otimes}+\boldsymbol{F}^{-\mathrm{t}} \underline{\otimes} \boldsymbol{F}^{-1}\right]+\lambda\left[\boldsymbol{F}^{-\mathrm{t}} \otimes \boldsymbol{F}^{-\mathrm{t}}-\log J \boldsymbol{F}^{-\mathrm{t}} \otimes \boldsymbol{F}^{-1}\right], \\
\overline{\mathbb{A}}:=\frac{\partial^{2} \bar{\psi}}{\partial \overline{\boldsymbol{F}}^{2}}= & \bar{\mu}\left[\boldsymbol{I} \bar{\otimes} \overline{\boldsymbol{I}}+\overline{\boldsymbol{F}}^{-\mathrm{t}} \underline{\otimes} \overline{\boldsymbol{F}}^{-1}-\left[\overline{\boldsymbol{F}} \cdot \overline{\boldsymbol{F}}^{-1}\right] \bar{\otimes}\left[\overline{\boldsymbol{F}}^{-1} \cdot \overline{\boldsymbol{F}}^{-\mathrm{t}}\right]\right] \\
& +\bar{\lambda}\left[\overline{\boldsymbol{F}}^{-\mathrm{t}} \otimes \overline{\boldsymbol{F}}^{-\mathrm{t}}-\log \bar{J}\left[\overline{\boldsymbol{F}}^{-\mathrm{t}} \otimes \overline{\boldsymbol{F}}^{-1}-\left[\overline{\boldsymbol{F}} \cdot \overline{\boldsymbol{F}}^{-1}\right] \bar{\otimes}\left[\overline{\boldsymbol{F}}^{-1} \cdot \overline{\boldsymbol{F}}^{-\mathrm{t}}\right]\right]\right] .
\end{aligned}
$$

Unlike the macroscopic deformation gradient and the macroscopic Piola stress, macroscopic Piola tangent ${ }^{\mathrm{M}} \mathbb{A}$ does not follow from the volume average of its microscopic counterpart in the bulk and on the surface. This is of particular importance in full $\mathrm{FE}^{2}$ homogenisation. The macroscopic Piola tangent reads

$$
{ }^{\mathrm{M}} \mathbb{A}=\frac{1}{\mathscr{V}_{0}} \int_{\mathcal{B}_{0}} \mathbb{A}+\mathbb{A}: \mathbb{B} \mathrm{d} V+\frac{1}{\mathscr{V}_{0}} \int_{\mathcal{I}_{0}} \overline{\mathbb{A}}+\overline{\mathbb{A}}: \mathbb{B} \mathrm{d} A
$$

in which the fourth-order tensor $\mathbb{B}$ denotes the linear mapping from $\delta^{\mathrm{M}} \boldsymbol{F}$ to its fluctuation $\delta \widetilde{\boldsymbol{F}}$ as $\delta \widetilde{\boldsymbol{F}}=\mathbb{B}: \delta^{\mathrm{M}} \boldsymbol{F}$. Detailed derivation of this quantity is given in Appendix 3.

For the numerical examples presented in this section, the material parameters of the matrix are fixed as $\mu=8$ and $\lambda=12.16$. The ratio of the inclusion to matrix Lamé parameters, denoted incl./matr., varies though. For instance, when incl./matr. $=10$, the inclusion material is 10 times stiffer than the matrix, and when incl./matr. $=0.1$, the inclusion material is 10 times more compliant than the matrix. The Poisson ratio for both the inclusion and the matrix is $v=0.3$. The interface material parameter is chosen as $\bar{\mu}=0.2$ for all the examples unless it is explicitly mentioned. For further details on interface material parameters, see [79, 80], among others. Different sizes of microstructures are considered. Here, the 

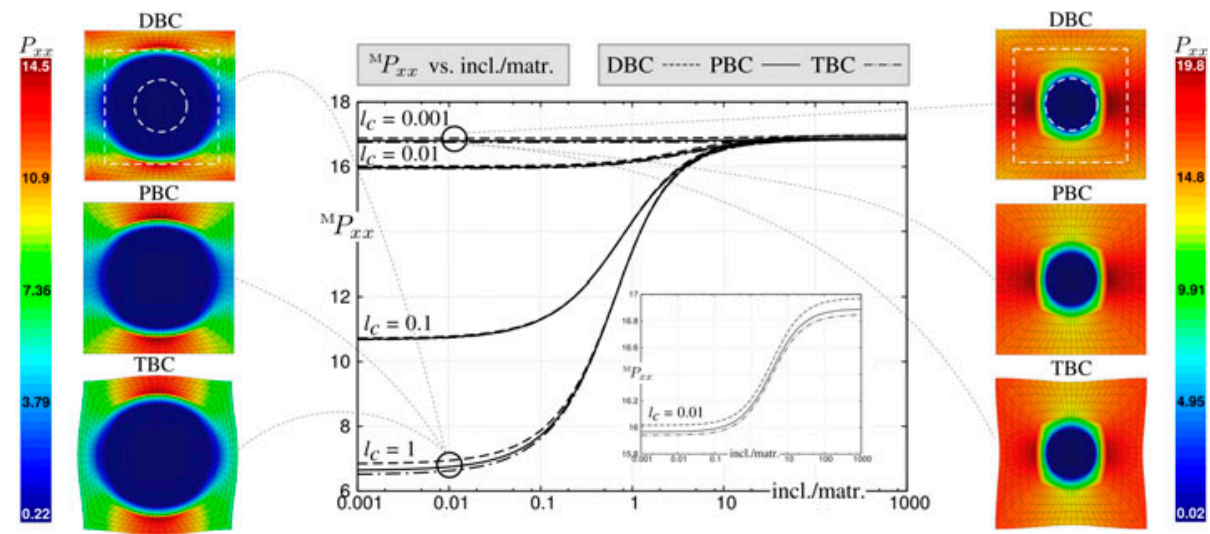

Figure 3. (colour online) $x x$-component of the macroscopic Piola stress with increasing the value of incl./matr. for two-dimensional unit-cells with $I_{c}=1,0.1,0.01$ and 0.001 which undergo $50 \%$ of volumetric expansion. The distributions of the microscopic Piola stress within the microstructure for incl./matr. $=0.01$ when $I_{c}=1$ (left) and $I_{c}=0.001$ (right) are depicted next to the plot.

term 'size' refers to the characteristic length of the RVE and is denoted $l_{c}$. Clearly, larger values of $l_{c}$ correspond to larger microstructures and vice versa. For all the following numerical examples, the inclusion volume fraction is always constant and assumed to be $f=25 \%$. Moreover, the geometries of all the samples are discretised using bilinear bulk and linear interface elements for two-dimensional examples and trilinear bulk and bilinear interface elements for three-dimensional examples.

\subsection{Influence of the material ratio}

In this section, we investigate on the influence of the inclusion to matrix stiffness ratio on the overall response of microstructures accounting for elastic interfaces. Different values of incl./matr. ranging from 0.001 with inclusions resembling voids up to 1000 with inclusion resembling rigid bodies are considered. We prescribe a volumetric expansion of $50 \%$ via the macroscopic deformation gradient on the micro-problem and evaluate the corresponding macroscopic Piola stress via relation $(4)_{2}$. This choice is made mainly due to the fact that under volumetric expansion, the interface effects are more pronounced compared to other deformation types. In order to solve the boundary value problem, DBC, $\mathrm{PBC}$ and $\mathrm{TBC}$ are utilised. In addition, we consider four microstructures with different sizes of $l_{c}=1,0.1,0.01$ and 0.001 .

Figure 3 shows that for larger microstructures, increasing the stiffness ratio from 0.001 to 0.1 does not have a significant influence on the overall response of the microstructure. However, increasing the stiffness ratio from 0.1 to 10 leads to a rapid rise of the macroscopic response. Further increase of the stiffness ratio does not influence the overall response considerably. Variation of the macroscopic response with increasing the stiffness ratio is less significant for smaller 
microstructures. Particularly, for $l_{c}=0.001$, the interface effects dominate the mechanism of the microstructure to such an extent that the material properties of the inclusion do not matter anymore, and almost the same results are obtained for all values of the material ratio. That is, elastic interfaces surrounding the inclusions play the role of a cloak of invisibility and do not let the microstructure feel the existence of the inclusions. At this point, the macroscopic response of the microstructure is saturated and reaches an upper bound beyond which 'smaller is stronger' trend is no longer significant. A similar argument holds for inclusions. That is, for all values of $l_{c}$, the macroscopic response is left almost unaffected for incl./matr. larger than 100 and further stiffening of the inclusions compared to the matrix does not lead to any stiffer macroscopic response. Moreover, it is observed that regardless of the material ratio and size of the microstructure, $\mathrm{DBC}$ and $\mathrm{TBC}$ provide the stiffest and the most compliant overall response, respectively. The results obtained via $\mathrm{PBC}$ lie between the ones associated with $\mathrm{DBC}$ and TBC. This observation is in accordance with what is reported for classical computational homogenisation. Another interesting point is that for larger microstructures where the interface effect is almost negligible, results of the different boundary conditions coincide when incl./matr. $=1$. That is to be expected as the interface contribution is minor and the microstructure is nearly homogeneous. However, for smaller microstructures, where the interface effect is not negligible anymore, the microstructure does not resemble a homogeneous structure even for incl./matr. = 1 and thus, the gap between the results of the different boundary conditions becomes more noticeable. This fact is illustrated in a subplot in Figure 3. The deformations of the microstructures as well as the distribution of the $x x$-component of the microscopic Piola stress for incl./matr $=$ 0.01 obtained from DBC, PBC and TBC are depicted in the plot. The deformed microstructures on the left correspond to a microstructure with $l_{c}=1$ and the ones on the right correspond to a microstructure with $l_{c}=0.001$. Clearly, in smaller microstructures, interface effects are so strong that the interface preserves its initial configuration and the portion of the body surrounded by interfaces remains nearly undeformed even when the inclusions are 100 times more compliant to the matrix.

In order to illustrate the performance of the presented framework for threedimensional problems, a similar numerical study is carried out for a cubic microstructure containing a spherical inclusion at its centre with the inclusion volume fraction $f=25 \%$. For this set of numerical examples, two microstructures with $l_{c}=1$ and 0.001 are considered. The distributions next to the plot in Figure 4 illustrate the greater resistance of the interface against deformation for smaller microstructures. It is also verified that the differences between the results of the boundary conditions are larger compared to the two-dimensional examples. Overall, the evolution pattern of macroscopic response of the threedimensional microstructure with respect to incl./matr. is similar to the one obtained for the two-dimensional microstructure. 

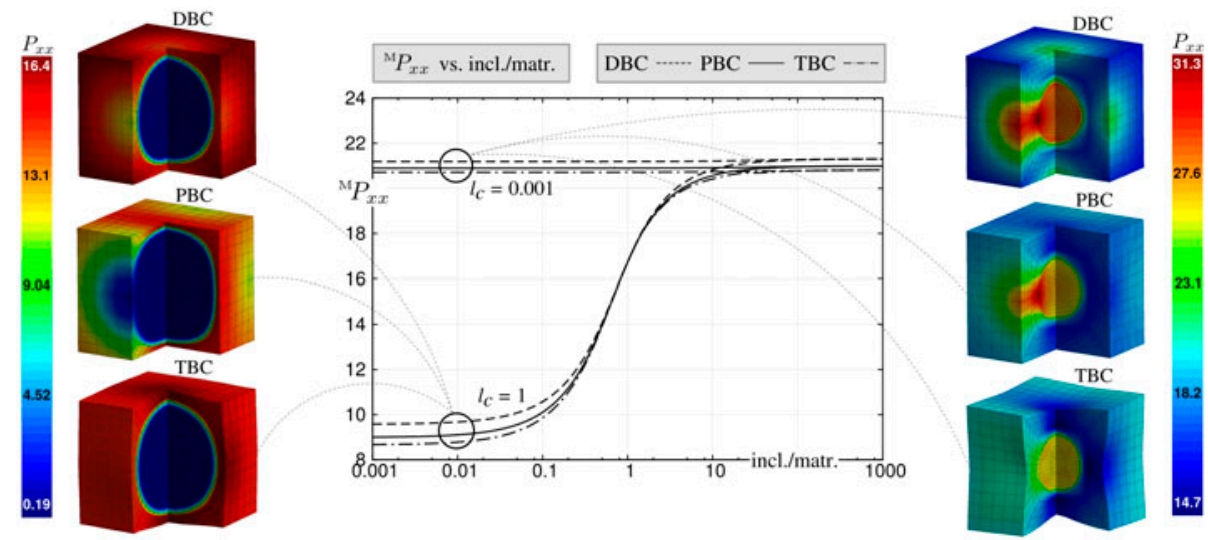

Figure 4. (colour online) $x x$-component of the macroscopic Piola stress with increasing the value of incl./matr. for three-dimensional unit-cells with $I_{c}=1$ and 0.001 which undergo $50 \%$ of volumetric expansion. The distributions of the microscopic Piola stress within the microstructure for incl./matr. $=0.01$ when $I_{c}=1$ (left) and $I_{c}=0.001$ (right) are depicted next to the plot.

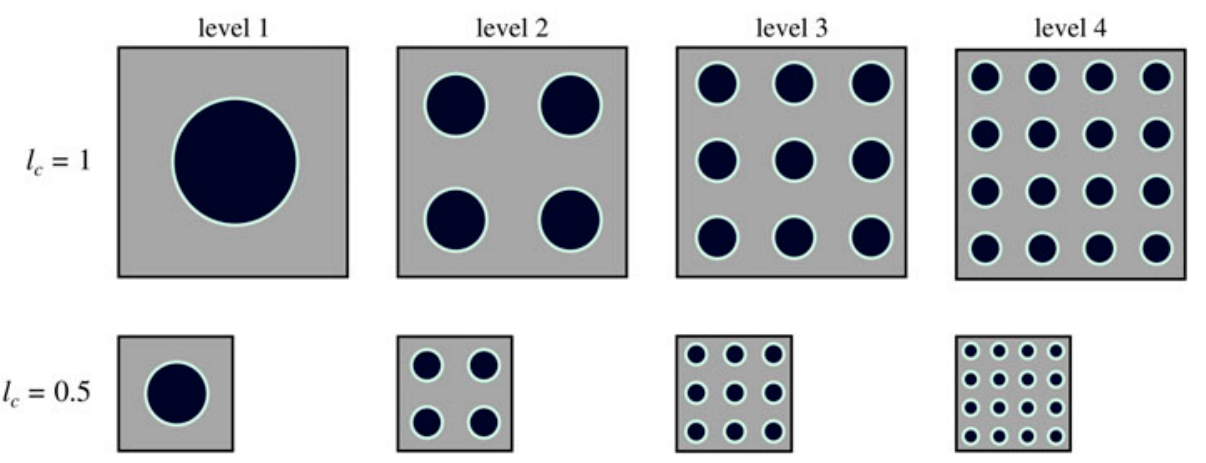

Figure 5. (colour online) Illustration of the size and the level of periodic RVEs. Different levels refer to the microstructures of different materials with different inclusion arrangements.

\subsection{Influence of the number of inclusions}

This section details on the macroscopic response when the number of inclusions within the microstructure increases while the size and the inclusion volume fraction are kept constant, see Figure 5. The inclusions are added such that the arrangement of the inclusions remains always periodic or more precisely, uniformly ordered. Note, the different levels in Figure 5 refer to the microstructures of different materials and determining the size of the RVE is not the purpose of this section.

For the following numerical examples, we prescribe a volume expansion of $25 \%$ on the microstructures and employ $\mathrm{DBC}, \mathrm{PBC}$ and $\mathrm{TBC}$ to evaluate the resulting macroscopic stresses. Six different sizes of $l_{c}=100,10,1,0.1,0.01$ and 0.001 as well as three different inclusions to matrix stiffness ratio of incl./matr. $=$ $0.1,1$ and 10 for each size of the microstructure are considered. 


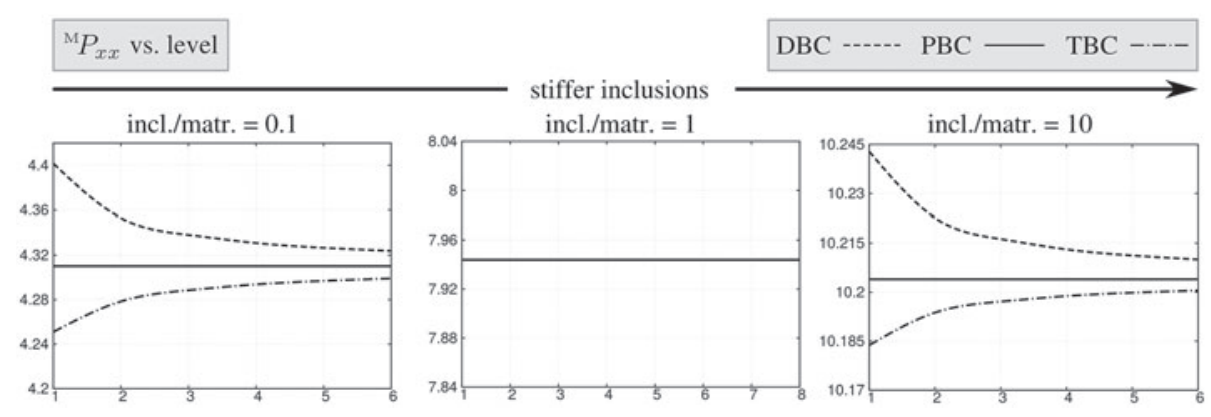

Figure 6. $x x$-component of the macroscopic Piola stress versus level of RVE for incl./matr. $=0.1,1$ and 10 when elastic interfaces are not included. The results from different boundary conditions get closer to each other as the level increases. Different boundary conditions render similar results when incl./matr. $=1$.

First, we investigate the case in which $\bar{\mu}=0$ equivalent to having no interface elasticity, at all. The numerical results depicted in Figure 6 show that regardless of the material ratio, the effective macroscopic property obtained using PBC remain constant for all the levels. This is to be expected since the inclusions are arranged periodically and the area-to-volume ratio does not influence the overall response. Clearly, when incl./matr. $=1, \mathrm{DBC}, \mathrm{TBC}$ and $\mathrm{PBC}$ furnish identical results. However, when the inclusion and the matrix are not of the same type, the results of the $\mathrm{DBC}$ and TBC get closer to the results of the PBC from the top and from the bottom, respectively, as the number of inclusions within the microstructure increases. Obviously, when elastic interfaces are excluded, no size effect is captured in the material response and changing the size of the microstructure does not influence the results.

In the next step, we set the interface material property to $\bar{\mu}=0.2$ and conduct the same study for microstructures of different sizes. The left column of Figure 7 illustrates the results for the microstructures in which the inclusions are 10 times more compliant to the matrix. The numerical results show that even for very large microstructures, i.e. $l_{c}=100$, the results from different boundary conditions are influenced by the presence of the interfaces and the effective property rises slightly as the level of the microstructure increases. This is mainly due to larger area-to-volume ratio of higher levels and as a result, larger interface effects on the material response. Interface effects can be further amplified by reducing the size of the microstructure leading to a stiffer overall response. For instance, when $l_{c}=10$, starting from the level 2, DBC provide stiffer response for higher levels which is in contrast to what is observed when the interface effects are neglected. For smaller microstructures, the macroscopic property increases monotonically as the level of the microstructure increases for all three types of the boundary conditions. Clearly, the difference between the macroscopic values obtained for $\bar{\mu}=0$ (illustrated by grey colour) and $\bar{\mu}=0.2$ becomes larger as the level of the microstructure increases and the size of the microstructure decreases. 


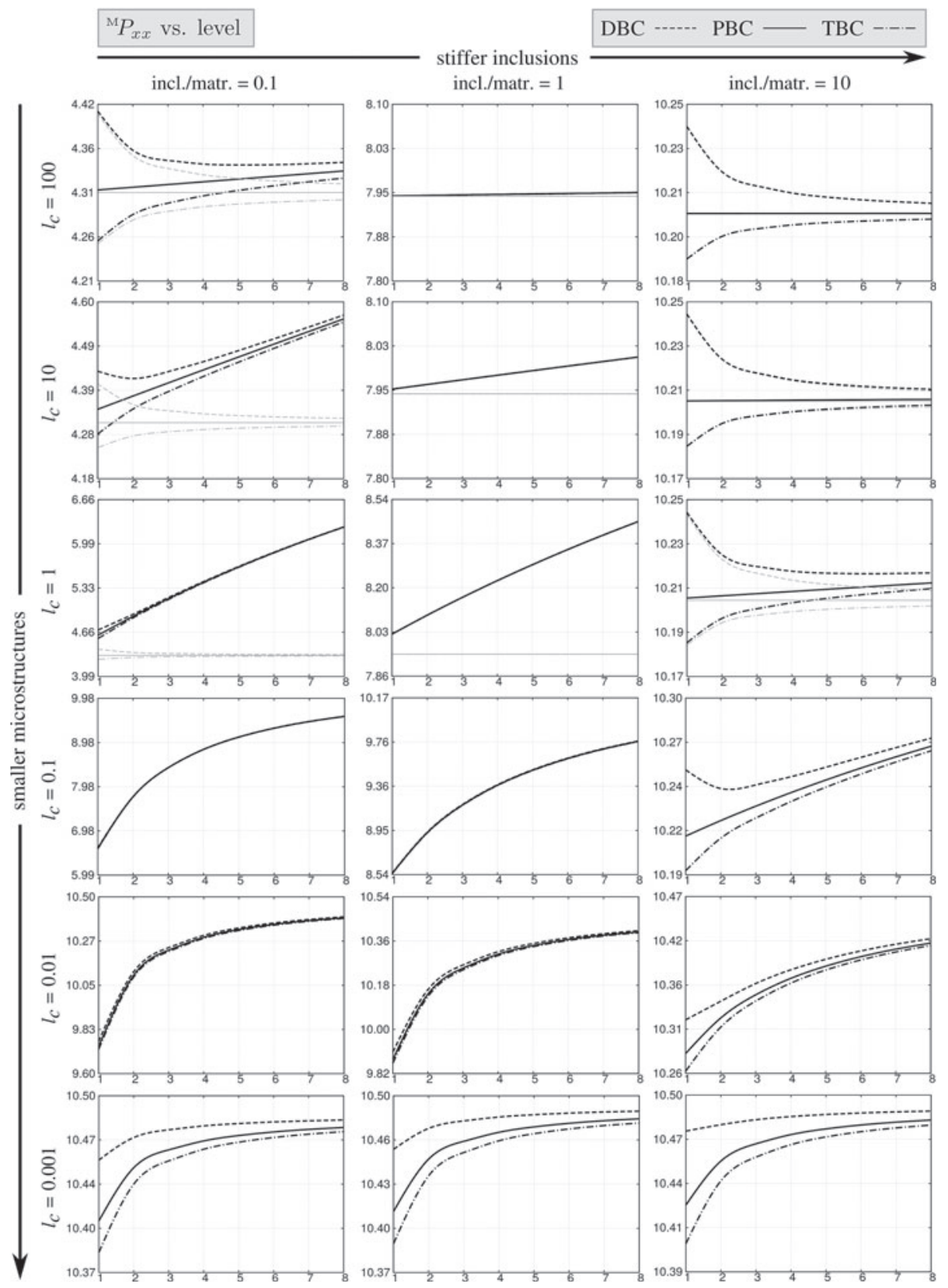

Figure 7. Macroscopic property versus level of periodic microstructures for incl./matr. $=0.1,1$ and 10 and different sizes when elastic interfaces are included and $\bar{\mu}=0.2$. The grey lines indicate the results for classical first-order computational homogenization.

The middle column of Figure 7 depicts the results for the microstructures where the inclusions and the matrix are identical. As anticipated, the overall response of the microstructures is essentially stiffer compared to the case that incl./matr. $=0.1$. Numerical results indicate that the macroscopic response becomes stiffer as the level increases for all sizes of the microstructure. In analogy 
to what is reported in Section 4.1, different boundary conditions render almost identical macroscopic response when the size of the microstructure is equal or larger than 0.1 which is expected to be observed for homogeneous materials. However, the difference between the results of different boundary conditions becomes larger for smaller microstructures.

The right column of Figure 7 shows the results of the same numerical studies for the case that the inclusions are 10 times stiffer than the matrix. It is verified that the interface effects are cancelled altogether by the stiff inclusions for microstructure with $l_{c}=100$ and $l_{c}=10$. Nevertheless, in microstructures smaller than $l_{c}=10$, interface effects become strong enough to overlay the stiff inclusions. Similar to what is observed for incl./matr. $=0.1$, for microstructures smaller than a certain $l_{c}$, here $l_{c}=0.1$, the macroscopic Piola stress increases monotonically as the level of the microstructure increases for all three types of the boundary conditions.

Note, when the size of the microstructure is very small, the macroscopic responses obtained from all the three types of boundary conditions become independent of the material properties of the inclusion, and almost same results are obtained for incl./matr. $=0.1,1$ and 10. At this limit, the overall response of the microstructure is fully saturated and the material response reaches its upper bound.

Moreover, the numerical results confirm the existence of bounds for interfaceenhanced computational homogenisation. It is observed that DBC and TBC provide upper and lower bounds for the size-dependent behaviour of the material response when energetic interfaces are taken into account. This observation extends the commonly accepted notion of bounds in first-order classical computational homogenisation. Figure 8 illustrates the macroscopic response of several microstructures when $l_{c}=10$ and incl./matr. $=0.1$ for two cases in which elastic interfaces are either considered (black curves) or neglected (grey curves). Clearly, the highlighted area between the curves represents the range of admissible macroscopic responses recovered by employing different boundary conditions such as PBC or mixed type boundary conditions.

\section{Comparing numerical and analytical results}

The goal of this section is to compare the numerical results against the analytical solution associated with small strain elasticity. The analytical solution to evaluate the effective bulk and shear modulus of a circular RVE containing a circular inclusion at its centre associated with a unidirectional fibre composite is given by $[81,82]$. Extension of the analytical solution to account for elastic interfaces is developed in [14,83-85], among others. In this section, we employ the formulations based on the composite cylinder approach to compute the effective in-plane bulk and shear modulus when the interfaces are taken into account. The effective in-plane bulk modulus reads 


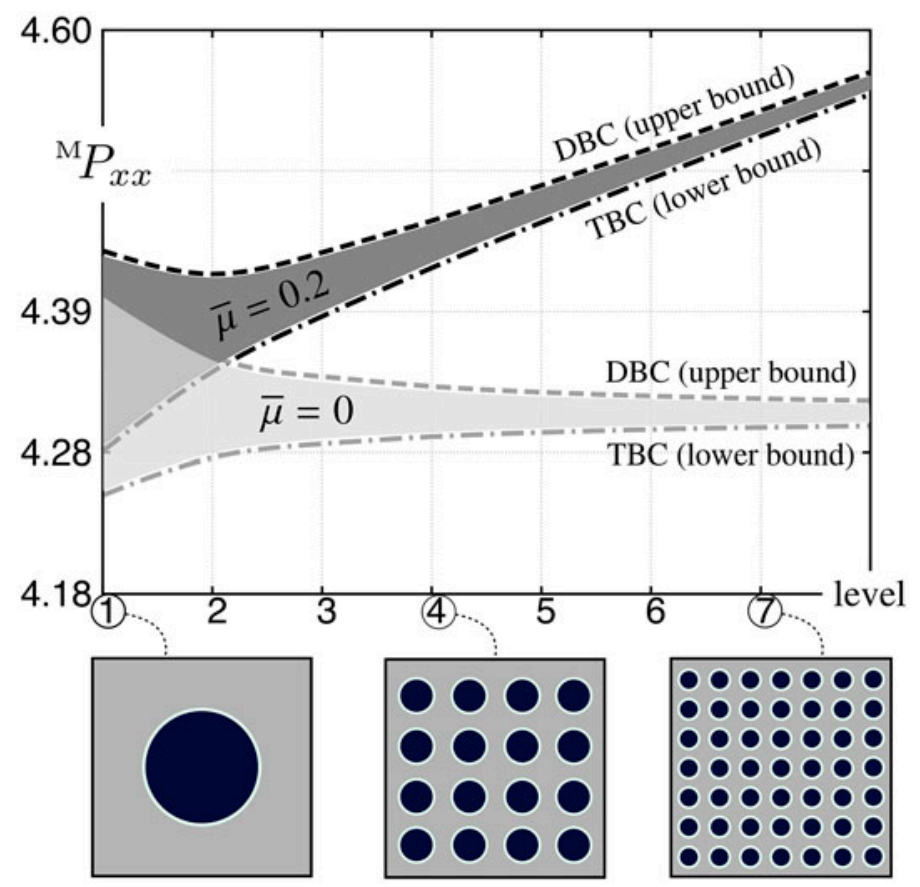

Figure 8. (colour online) Illustration of bounds for interface-enhanced computational homogenisation capturing size-effects.

$$
\begin{aligned}
\mathrm{M}_{\kappa}=\lambda^{\mathrm{m}}+\mu^{\mathrm{m}}+\frac{f}{\chi} \quad \text { with } \quad \chi= & \frac{r}{r\left[\lambda^{\mathrm{i}}+\mu^{\mathrm{i}}\right]-r\left[\lambda^{\mathrm{m}}+\mu^{\mathrm{m}}\right]+\bar{\mu}} \\
& +\frac{1-f}{\lambda^{\mathrm{m}}+2 \mu^{\mathrm{m}}},
\end{aligned}
$$

in which $f$ and $r$ denote the inclusion's volume fraction and the radius. Moreover, $\{\bullet\}^{\mathrm{m}}$ and $\{\bullet\}^{\mathrm{i}}$ represent quantities in the matrix and the inclusion, respectively. Unlike for the effective bulk modulus, a simple closed form explicit solution for the effective shear modulus ${ }^{\mathrm{M}} \mu$ does not exist. The details of the derivation of the analytical solution to compute the effective shear modulus is given in [14], among others. Computational aspects of interface-enhanced computational homogenisation in the context of small strain are fundamentally similar to the finite strain setting and are not discussed here for the sake of space. Numerical simulations are realised over a square RVE with a single circular inclusion at its centre with volume fraction $f=25 \%$. Similar to the previous sections, DBC, $\mathrm{PBC}$ and TBC are utilised to solve the micro-problem.

Figure 9 depicts the effective in-plane bulk and shear modulus with respect to size for various values of incl./matr. and interface material parameter $\bar{\mu}$. It is observed that different boundary conditions and analytical solutions render almost similar results in estimating the effective in-plane bulk modulus for all the values of incl./matr. and $\bar{\mu}$. The results confirm that no size effect is observed when $\bar{\mu}$ is set to zero. However, when elastic interfaces are taken into account, 

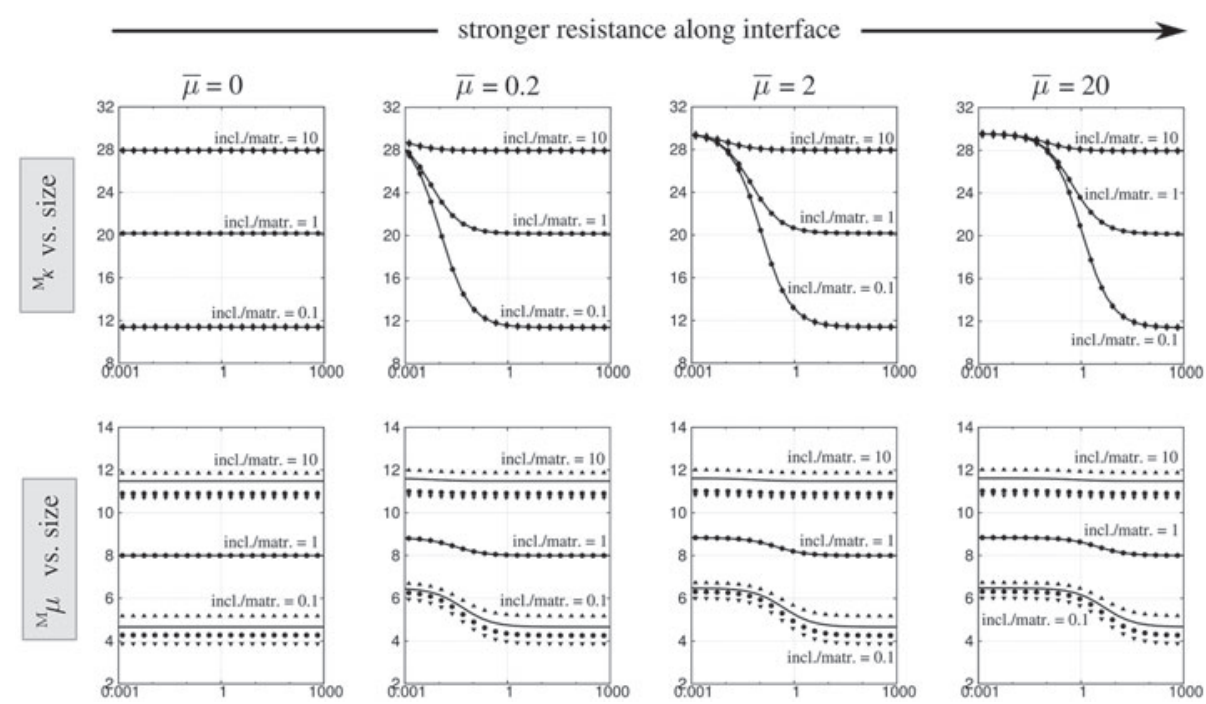

Figure 9. Comparison between numerical results obtained from $\mathrm{DBC}(\boldsymbol{\Delta}), \mathrm{PBC}(\bullet), \mathrm{TBC}(\boldsymbol{\nabla})$ and analytical solution (solid lines) for the effective bulk (top) and shear modulus (bottom) with respect to size of the microstructure.

the effective in-plane bulk modulus starts to increase when the microstructure is small enough and eventually approaches its upper bound as the size of the microstructure decreases further. Obviously, the convergence of the overall response to the upper bound occurs at larger size of the microstructure when $\bar{\mu}$ increases.

In the case of the effective shear modulus, the results from different boundary conditions deviate from each other for all values of $\bar{\mu}$. It is observed that the analytical solution always lies between the results from DBC and PBC and accordingly between DBC and TBC. Note, unlike the bulk modulus, the shear moduli obtained for different incl./matr. do not converge to each other as the size decreases. More interestingly, it is observed that the analytical solution provides closer results to the numerical ones for smaller microstructures in which interface effects are more pronounced. This is justified by the fact that the volumetric expansion requires a change of the length of the interface. In other words, a volumetric expansion cannot be prescribed without extending the interface. But this is not the case for the shear. That is, the length of the interface may remain unchanged under a shear deformation. Overall, the analytical solution captures the trend for the effective shear modulus almost perfectly from a qualitative point of view and only with a shift from the numerical solutions for incl./matr $=0.1$ and 10 .

\section{Conclusion}

A systematic study to compute the bounds on the overall response of a microstructure including elastic interfaces is presented. The computational 
homogenisation framework accounting for interfaces captures the size-dependent effective behaviour of the material. The interface effects lead to the introduction of two relative length scales missing in classical first-order computational homogenisation. A generalised Hill-Mandel condition is formulated and suitable boundary conditions of the micro-scale problem are derived. The bounds on the overall response of a microstructure due to various boundary conditions are investigated. Furthermore, changing the size of the microstructure furnishes additional bounds on the overall response. Based on the numerical examples, we conclude that the microstructures at a certain size approach an upper bound for the material response, and thus, the widely recognised 'smaller is stronger' trend is no longer observed. In analogy to classical first-order computational homogenisation, $\mathrm{DBC}$ and $\mathrm{TBC}$ provide the stiffest and the most compliant response, respectively, while the results of the $\mathrm{PBC}$ lie between the results of the other two. In summary, the presented scheme allows us to better understand and predict the overall response of composites under various circumstances. This generic framework is broadly applicable to deepen our understanding of the size-dependent behaviour of materials with a large variety of applications in nano-structures.

\section{Disclosure statement}

No potential conflict of interest was reported by the authors.

\section{Funding}

The support of this work by the Cluster of Excellence 'Engineering of Advanced Materials' at the University of Erlangen-Nuremberg, funded by the DFG within the framework of its 'Excellence Initiative', is greatly appreciated.

\section{ORCID}

A. Javili (D) http://orcid.org/0000-0001-7965-7088

\section{References}

[1] K. Knowles and D. Smith, The application of surface dislocation theory to the f.c.c.-b.c.c. interface, Acta Crystallogr. A 38 (1982), pp. 34-40.

[2] P. Sharma and S. Ganti, Size-dependent Eshelby's tensor for embedded nano-inclusions incorporating surface/interface energies, J. Appl. Mech. 71 (2004), pp. 663-671.

[3] R. Dingreville, J. Qu, and M. Cherkaoui, Surface free energy and its effect on the elastic behavior of nano-sized particles, wires and films, J. Mech. Phys. Solids 53 (2005), pp. 1827-1854.

[4] W. Gao, S. Yu, and G. Huang, Finite element characterization of the size-dependent mechanical behaviour in nanosystems, Nanotechnology 17 (2006), pp. 1118-1122.

[5] H. Park and V. Laohom, Surface composition effects on martensitic phase transformations in nickel aluminium nanowires, Philos. Mag. 87 (2007), pp. 2159-2168. 
[6] S. Mogilevskaya, S. Crouch, and H. Stolarski, Multiple interacting circular nanoinhomogeneities with surface/interface effects, J. Mech. Phys. Solids 56 (2008), pp. 22982327.

[7] Y. Cheng and M. Verbrugge, The influence of surface mechanics on diffusion induced stresses within spherical nanoparticles, J. Appl. Phys. 104 (2008), p. 083521.

[8] J. Yvonnet, H. Quang, and Q.C. He, An XFEM/level set approach to modelling surface/interface effects and to computing the size-dependent effective properties of nanocomposites, Comput. Mech. 42 (2008), pp. 119-131.

[9] Z. Wang, Y. Zhao, and Z. Huang, The effects of surface tension on the elastic properties of nano structures, Int. J. Eng. Sci. 48 (2010), pp. 140-150.

[10] R. Ansari and S. Sahmani, Bending behavior and buckling of nanobeams including surface stress effects corresponding to different beam theories, Int. J. Eng. Sci. 49 (2011), pp. 12441255.

[11] H. Altenbach and V. Eremeyev, On the shell theory on the nanoscale with surface stresses, Int. J. Eng. Sci. 49 (2011), pp. 1294-1301.

[12] G. Chatzigeorgiou, A. Javili, and P. Steinmann, Interface properties influence the effective dielectric constant of composites, Philos. Mag. 95 (2015), pp. 3402-3412.

[13] N. Cordero, S. Forest, and E. Busso, Second strain gradient elasticity of nano-objects, J. Mech. Phys. Solids 97 (2016), pp. 92-124.

[14] G. Chatzigeorgiou, F. Meraghni, and A. Javili, Generalized interfacial energy and size effects in composites, J. Mech. Phys. Solids 106 (2017), pp. 257-282.

[15] K. Kiani, Exact postbuckling analysis of highly stretchable-surface energetic-elastic nanowires with various ends' conditions, Int. J. Mech. Sci. 124-125 (2017), pp. 242-252.

[16] H. Duan, J. Wang, and B. Karihaloo, Theory of elasticity at the nanoscale, Adv. Appl. Math. 42 (2009), pp. 1-68.

[17] A. Javili, A. McBride, and P. Steinmann, Thermomechanics of solids with lowerdimensional energetics: On the importance of surface, interface, and curve structures at the nanoscale. a unifying review, Appl. Mech. Rev. 65 (2013), p. 010802.

[18] G. Moeckel, Thermodynamics of an interface, Arch. Ration. Mech. Anal. 57 (1975), pp. 255-280.

[19] A. Murdoch, A thermodynamical theory of elastic material interfaces, Q. J. Mech. Appl. Math. 29 (1976), pp. 245-275.

[20] F. Dell'isola and A. Romano, On the derivation of thermomechanical balance equations for continuous systems with a nonmaterial interface, Int. J. Eng. Sci. 25 (1987), pp. 14591468.

[21] E. Fried and M. Gurtin, Thermomechanics of the interface between a body and its environment, Continuum Mech. Thermodyn. 19 (2007), pp. 253-271.

[22] A. Javili, N. Ottosen, M. Ristinmaa, and J. Mosler, Aspects of interface elasticity theory, Math. Mech. Solids (2017). doi: 10.1177/1081286517699041.

[23] M. Gurtin and A. Murdoch, A continuum theory of elastic material surfaces, Arch. Ration. Mech. Anal. 57 (1975), pp. 291-323.

[24] P. Steinmann, On boundary potential energies in deformational and configurational mechanics, J. Mech. Phys. Solids 59 (2008), pp. 772-800.

[25] T. Chen, M. Chiu, and C. Weng, Derivation of the generalized young-laplace equation of curved interfaces in nanoscaled solids, J. Appl. Phys. 100 (2006), p. 074308.

[26] K. Park and G. Paulino, Cohesive zone models: A critical review of traction-separation relationships across fracture surfaces, Appl. Mech. Rev. 64 (2013), p. 060802.

[27] R. Dingreville, A. Hallil, and S. Berbenni, From coherent to incoherent mismatched interfaces: A generalized continuum formulation of surface stresses, J. Mech. Phys. Solids 72 (2014), pp. 40-60. 
[28] A. Javili, Variational formulation of generalized interfaces for finite deformation elasticity, Math. Mech. Solids (2017). doi: 10.1177/1081286517719938.

[29] D. Steigmann and R. Ogden, Plane deformations of elastic solids with intrinsic boundary elasticity, Proc. Royal Soc. A 453 (1997), pp. 853-877.

[30] D. Steigmann and R. Ogden, Elastic surface-substrate interactions, Proc. Royal Soc. A 455 (1999), pp. 437-474.

[31] P. Chhapadia, P. Mohammadi, and P. Sharma, Curvature-dependent surface energy and implications for nanostructures, J. Mech. Phys. Solids 59 (2011), pp. 2103-2115.

[32] X. Gao, Z. Huang, J. Qu, and D. Fang, A curvature-dependent interfacial energy-based interface stress theory and its applications to nano-structured materials: (i) general theory, J. Mech. Phys. Solids 66 (2014), pp. 59-77.

[33] P. Kanouté, D. Boso, J. Chaboche, and B. Schrefler, Multiscale methods for composites: A review, Arch. Comput. Methods Eng. 16 (2009), pp. 31-75.

[34] N. Charalambakis, Homogenization techniques and micromechanics. A survey and perspectives, Appl. Mech. Rev. 63 (2010), p. 030803.

[35] S. Saeb, P. Steinmann, and A. Javili, Aspects of computational homogenization at finite deformations: A unifying review from reuss' to voigt's bound, Appl. Mech. Rev. 68 (2016), p. 050801.

[36] K. Matouš, M. Geers, V. Kouznetsova, and A. Gillman, A review of predictive nonlinear theories for multiscale modeling of heterogeneous materials, J. Comput. Phys. 330 (2017), pp. 192-220.

[37] C. Miehe and A. Koch, Computational micro-to-macro transitions of discretized microstructures undergoing small strains, Arch. Appl. Mech. 72 (2002), pp. 300-317.

[38] P. Guidault, O. Allix, L. Champaney, and J. Navarro, A two-scale approach with homogenization for the computation of cracked structures, Comput. Struct. 85 (2007), pp. 1360-1371.

[39] M. Hain and P. Wriggers, Computational homogenization of micro-structural damage due to frost in hardened cement paste, Finite Elem. Anal. Des. 44 (2008), pp. 233-244.

[40] H. Inglis, P. Geubelle, and K. Matouš, Boundary condition effects on multiscale analysis of damage localization, Philos. Mag. 88 (2008), pp. 2373-2397.

[41] İ. Temizer and P. Wriggers, On the computation of the macroscopic tangent for multiscale volumetric homogenization problems, Comput. Methods in Appl. Mech. Eng. 198 (2008), pp. 495-510.

[42] I. Özdemir, W. Brekelmans, and M. Geers, $F E^{2}$ computational homogenization for the thermo-mechanical analysis of heterogeneous solids, Comput. Methods Appl. Mech. Eng. 198 (2008), pp. 602-613.

[43] M. Geers, V. Kouznetsova, and W. Brekelmans, Multi-scale computational homogenization: Trends and challenges, J. Comput. Appl. Math. 234 (2010), pp. 21752182.

[44] K. Terada, M. Kurumatani, T. Ushida, and N. Kikuchi, A method of two-scale thermomechanical analysis for porous solids with micro-scale heat transfer, Comput. Mech. 46 (2010), pp. 269-285.

[45] F. Larsson, K. Runesson, S. Saroukhani, and R. Vafadari, Computational homogenization based on a weak format of micro-periodicity for RVE-problems, Comput. Methods in Appl. Mech. Eng. 200 (2011), pp. 11-26.

[46] E. Coenen, V. Kouznetsova, E. Bosco, and M. Geers, A multi-scale approach to bridge microscale damage and macroscale failure: a nested computational homogenizationlocalization framework, Int. J. Fract. 178 (2012), pp. 157-178.

[47] D. Kochmann and G. Venturini, Homogenized mechanical properties of auxetic composite materials in finite-strain elasticity, Smart Mater. Struct. 22 (2013), p. 084004. 
[48] A. Javili, G. Chatzigeorgiou, and P. Steinmann, Computational homogenization in magneto-mechanics, Int. J. Solids Struct. 50 (2013), pp. 4197-4216.

[49] D. Balzani, L. Scheunemann, D. Brands, and J. Schröder, Construction of two- and threedimensional statistically similar RVEs for coupled micro-macro simulations, Comput. Mech. 54 (2014), pp. 1269-1284.

[50] G. Chatzigeorgiou, A. Javili, and P. Steinmann, Unified magnetomechanical homogenization framework with application to magnetorheological elastomers, Math. Mech. Solids 19 (2014), pp. 193-211.

[51] E. Bosco, V. Kouznetsova, and M. Geers, Multi-scale computational homogenizationlocalization for propagating discontinuities using X-FEM, Int. J. Numer. Methods Eng. 102 (2015), pp. 496-527.

[52] Y. Cong, S. Nezamabadi, H. Zahrouni, and J. Yvonnet, Multiscale computational homogenization of heterogeneous shells at small strains with extensions to finite displacements and buckling, Int. J. Numer. Methods Eng. 104 (2015), pp. 235-259.

[53] C. Miehe, J. Schröder, and J. Schotte, Computational homogenization analysis in finite plasticity Simulation of texture development in polycrystalline materials, Comput. Methods in Appl. Mech. Eng. 171 (1999), pp. 387-418.

[54] F. Feyel and J.L. Chaboche, $F E^{2}$ multiscale approach for modelling the elastoviscoplastic behaviour of long fibre SiC/Ti composite materials, Comput. Methods in Appl. Mech. Eng. 183 (2000), pp. 309-330.

[55] V. Kouznetsova, W. Brekelmans, and F. Baaijens, An approach to micro-macro modeling of heterogeneous materials, Comput. Mech. 27 (2001), pp. 37-48.

[56] K. Terada, I. Saiki, K. Matsui, and Y. Yamakawa, Two-scale kinematics and linearization for simultaneous two-scale analysis of periodic heterogeneous solids at finite strain, Comput. Methods Appl. Mech. Eng. 192 (2003), pp. 3531-3563.

[57] W. Drugan and J. Willis, A micromechanics-based non local constitutive equation and estimates of representative volume element size for elastic composites, J. Mech. Phys. Solids 44 (1996), pp. 497-524.

[58] T. Kanit, S. Forest, I. Galliet, V. Mounoury, and D. Jeulin, Determination of the size of the representative volume element for random composites: statistical and numerical approach, Int. J. Solids Struct. 40 (2003), pp. 3647-3679.

[59] M. Ostoja-Starzewski, Material spatial randomness: From statistical to representative volume element, Probab. Eng. Mech. 21 (2006), pp. 112-132.

[60] A. Javili, P. Steinmann, and J. Mosler, Micro-to-macro transition accounting for general imperfect interfaces, Comput. Methods in Appl. Mech. Eng. 317 (2017), pp. 274-317.

[61] D. Davydov, A. Javili, and P. Steinmann, On molecular statics and surface-enhanced continuum modeling of nano-structures, Comput. Mater. Sci. 69 (2013), pp. 510-519.

[62] H. Park, E. Karpov, K. Wing, and P. Klein, The bridging scale for two-dimensional atomistic/continuum coupling, Philos. Mag. 85 (2005), pp. 79-113.

[63] H. Park, P. Klein, and G. Wagner, A surface Cauchy-Born model for nanoscale materials, Int. J. Numer. Methods Eng. 68 (2006), pp. 1072-1095.

[64] V. Kouznetsova, M. Geers, and W. Brekelmans, Multi-scale second-order computational homogenization of multi-phase materials: A nested finite element solution strategy, Comput. Methods Appl. Mech. Eng. 193 (2004), pp. 5525-5550.

[65] R. Hill, On constitutive macro-variables for heterogeneous solids at finite strain, Proc. Royal Soc. A 326 (1972), pp. 131-147.

[66] G. Chatzigeorgiou, A. Javili, and P. Steinmann, Multiscale modelling for composites with energetic interface at the micro- or nanoscale, Math. Mech. Solids 20 (2013), pp. 11301145. 
[67] S. Hollister and N. Kikuchi, A comparison of homogenization and standard mechanic analyses for periodic porous composites, Comput. Mech. 10 (1992), pp. 73-95.

[68] M. Hori and S. Nemat-Nasser, On two micromechanics theories for determining micromacro relations in heterogeneous solids, Mech. Mater. 31 (1999), pp. 667-682.

[69] D. Perić and E. de Souza, Neto, R.A. Feijóo, M. Partovi, and A. Carneiro Molina, On micro-to-macro transitions for multi-scale analysis of non-linear heterogeneous materials: unified variational basis and finite element implementation, Int. J. Numer. Methods Eng. 87 (2011), pp. 149-170.

[70] L. Kaczmarczyk, C. Pearce, and N. Bićanić, Scale transition and enforcement of RVE boundary conditions in second-order computational homogenization, Int. J. Numer. Methods Eng. 74 (2008), pp. 506-522.

[71] M. Jiang, K. Alzebdeh, I. Jasiuk, and M. Ostoja-Starzewski, Scale and boundary conditions effects in elastic properties of random composites, Acta Mech. 148 (2001), pp. 63-78.

[72] İ. Temizer and T.I. Zohdi, A numerical method for homogenization in non-linear elasticity, Comput. Mech. 40 (2007), pp. 281-298.

[73] A. Javili, A. McBride, J. Mergheim, P. Steinmann, and U. Schmidt, Micro-to-macro transitions for continua with surface structure at the microscale, Int. J. Solids Struct. 50 (2013), pp. 2561-2572.

[74] A. Javili, G. Chatzigeorgiou, A. McBride, P. Steinmann, and C. Linder, Computational homogenization of nano-materials accounting for size effects via surface elasticity, GAMM-Mitt. 38 (2015), pp. 285-312.

[75] İ. Temizer and P. Wriggers, Homogenization in finite thermoelasticity, J. Mech. Phys. Solids 59 (2011), pp. 344-372.

[76] C. Miehe, Computational micro-to-macro transitions for discretized micro-structures of heterogeneous materials at finite strains based on the minimization of averaged incremental energy, Comput. Methods in Appl. Mech. Eng. 192 (2003), pp. 559-591.

[77] A. Javili, S. Saeb, and P. Steinmann, Aspects of implementing constant traction boundary conditions in computational homogenization via semi-dirichlet boundary conditions, Comput. Mech. 59 (2016), pp. 1-15.

[78] A. Javili, A. McBride, P. Steinmann, and B. Reddy, A unified computational framework for bulk and surface elasticity theory: a curvilinear-coordinate-based finite element methodology, Comput. Mech. 54 (2014), pp. 745-762.

[79] V. Shenoy, Atomistic calculations of elastic properties of metallic fcc crystal surfaces, Phys. Rev. B 71 (2005), p. 094104.

[80] A. Javili, A. McBride, P. Steinmann, and B. Reddy, Relationships between the admissible range of surface material parameters and stability of linearly elastic bodies, Philos. Mag. 92 (2012), pp. 3540-3563.

[81] Z. Hashin and B. Rosen, The elastic moduli of reinforced-reinforced materials, J. Appl. Mech. 31 (1964), pp. 223-232.

[82] R. Christensen and K. Lo, Solution for effective shear properties in three phase sphere and cylinder models, J. Mech. Phys. Solids 27 (1979), pp. 315-330.

[83] P. Sharma, S. Ganti, and N. Bhate, Effect of surfaces on the size-dependent elastic state of nano-inhomogeneities, Appl. Phys. Lett. 82 (2003), pp. 535-537.

[84] H. Duan, J. Wang, Z. Huang, and B. Karihaloo, Size-dependent effective elastic constants of solids containing nano-inhomogeneities with interface stress, J. Mech. Phys. Solids 53 (2005), pp. 1574-1596.

[85] H. Duan, X. Yi, Z. Huang, and J. Wang, A unified scheme for prediction of effective moduli of multiphase composites with interface effects. Part I: Theoretical framework, Mech. Mater. 39 (2007), pp. 81-93. 


\section{Appendix 1. Proof of the Lemma (6)}

In order to prove the Lemma (6)

$$
\frac{1}{\mathscr{V}_{0}} \int_{\mathcal{S}_{0}} \boldsymbol{t}_{0} \cdot \delta \boldsymbol{\varphi} \mathrm{d} A+\frac{1}{\mathscr{V}_{0}} \int_{\mathcal{L}_{0}} \overline{\boldsymbol{t}}_{0} \cdot \delta \overline{\boldsymbol{\varphi}} \mathrm{d} L=\frac{1}{\mathscr{V}_{0}} \int_{\mathcal{B}_{0}} \boldsymbol{P}: \delta \boldsymbol{F} \mathrm{d} V+\frac{1}{\mathscr{V}_{0}} \int_{\mathcal{I}_{0}} \overline{\boldsymbol{P}}: \delta \overline{\boldsymbol{F}} \mathrm{d} A,
$$

we utilise the following divergence theorems

$$
\begin{aligned}
& \int_{\mathcal{B}_{0}} \operatorname{Div}\{\bullet\} \mathrm{d} V=\int_{\mathcal{S}_{0}}\{\bullet\} \cdot \boldsymbol{N} \mathrm{d} A-\int_{\mathcal{I}_{0}} \llbracket\{\bullet\} \rrbracket \cdot \overline{\boldsymbol{N}} \mathrm{d} A, \\
& \int_{\mathcal{I}_{0}} \overline{\operatorname{Div}}\{\bullet\} \mathrm{d} A=\int_{\mathcal{L}_{0}}\{\bullet\} \cdot \widetilde{\boldsymbol{N}} \mathrm{d} L-\int_{\mathcal{I}_{0}} \bar{k}\{\bullet\} \cdot \overline{\boldsymbol{N}} \mathrm{d} A,
\end{aligned}
$$

in which $\bar{k}$ is twice the mean curvature of the interface in the refernce configuration. We start from the left-hand side as

$$
\begin{aligned}
& \frac{1}{\mathscr{V}_{0}} \int_{\mathcal{S}_{0}} \boldsymbol{t}_{0} \cdot \delta \boldsymbol{\varphi} \mathrm{d} A+\frac{1}{\mathscr{V}_{0}} \int_{\mathcal{L}_{0}} \overline{\boldsymbol{t}}_{0} \cdot \delta \overline{\boldsymbol{\varphi}} \mathrm{d} L \\
& =\frac{1}{\mathscr{V}_{0}} \int_{\mathcal{B}_{0}} \operatorname{Div}[\delta \boldsymbol{\varphi} \cdot \boldsymbol{P}] \mathrm{d} V+\frac{1}{\mathscr{V}_{0}} \int_{\mathcal{I}_{0}} \llbracket \delta \boldsymbol{\varphi} \cdot \boldsymbol{P} \rrbracket \cdot \overline{\boldsymbol{N}} \mathrm{d} A \\
& +\frac{1}{\mathscr{V}_{0}} \int_{\mathcal{I}_{0}} \overline{\operatorname{Div}}[\delta \overline{\boldsymbol{\varphi}} \cdot \overline{\boldsymbol{P}}] \mathrm{d} A+\frac{1}{\mathscr{V}_{0}} \int_{\mathcal{L}_{0}} \bar{k} \delta \overline{\boldsymbol{\varphi}} \cdot \underbrace{\overline{\boldsymbol{P}} \cdot \overline{\boldsymbol{N}}}_{=\mathbf{0}} \mathrm{d} L \\
& =\frac{1}{\mathscr{V}_{0}} \int_{\mathcal{B}_{0}} \delta \boldsymbol{\varphi} \cdot \underbrace{\operatorname{Div} \boldsymbol{P}}_{=\mathbf{0}} \mathrm{d} V+\frac{1}{\mathscr{V}_{0}} \int_{\mathcal{B}_{0}} \boldsymbol{P}: \operatorname{Grad} \delta \boldsymbol{\varphi} \mathrm{d} V+\frac{1}{\mathscr{V}_{0}} \int_{\mathcal{I}_{0}} \delta \overline{\boldsymbol{\varphi}} \cdot \underbrace{\llbracket \boldsymbol{P} \rrbracket \cdot \overline{\boldsymbol{N}}}_{=-\overline{\mathrm{Div}} \overline{\boldsymbol{P}}} \mathrm{d} A \\
& +\frac{1}{\mathscr{V}_{0}} \int_{\mathcal{I}_{0}} \delta \overline{\boldsymbol{\varphi}} \cdot \overline{\operatorname{Div}} \overline{\boldsymbol{P}} \mathrm{d} A+\frac{1}{\mathscr{V}_{0}} \int_{\mathcal{I}_{0}} \overline{\boldsymbol{P}}: \delta \overline{\boldsymbol{F}} \mathrm{d} A \\
& =\frac{1}{\mathscr{V}_{0}} \int_{\mathcal{B}_{0}} \boldsymbol{P}: \delta \boldsymbol{F} \mathrm{d} V+\frac{1}{\mathscr{V}_{0}} \int_{\mathcal{I}_{0}} \overline{\boldsymbol{P}}: \delta \overline{\boldsymbol{F}} \mathrm{d} A .
\end{aligned}
$$

\section{Appendix 2. Proof of the generalised Hill's Lemma}

In order to prove the generalised Hill's Lemma

$$
\begin{aligned}
& \frac{1}{\mathscr{V}_{0}} \int_{\mathcal{B}_{0}} \boldsymbol{P}: \delta \boldsymbol{F} \mathrm{d} V+\frac{1}{\mathscr{V}_{0}} \int_{\mathcal{I}_{0}} \overline{\boldsymbol{P}}: \delta \overline{\boldsymbol{F}} \mathrm{d} A-{ }^{\mathrm{M}} \boldsymbol{P}: \delta^{\mathrm{M}} \boldsymbol{F} \\
& \quad=\frac{1}{\mathscr{V}_{0}} \int_{\mathcal{S}_{0}}\left[\delta \boldsymbol{\varphi}-\delta^{\mathrm{M}} \boldsymbol{F} \cdot \boldsymbol{X}\right] \cdot \boldsymbol{t}_{0} \mathrm{~d} A+\frac{1}{\mathscr{V}_{0}} \int_{\mathcal{L}_{0}}\left[\delta \overline{\boldsymbol{\varphi}}-\delta^{\mathrm{M}} \boldsymbol{F} \cdot \overline{\boldsymbol{X}}\right] \cdot \overline{\boldsymbol{t}}_{0} \mathrm{~d} L,
\end{aligned}
$$

we start from the right-hand side and manipulate it until we remain with the left-hand side as

$$
\begin{aligned}
\frac{1}{\mathscr{V}_{0}} \int_{\mathcal{S}_{0}}\left[\delta \boldsymbol{\varphi}-\delta^{\mathrm{M}} \boldsymbol{F} \cdot \boldsymbol{X}\right] \cdot \boldsymbol{t}_{0} \mathrm{~d} A+\frac{1}{\mathscr{V}_{0}} \int_{\mathcal{L}_{0}}\left[\delta \overline{\boldsymbol{\varphi}}-\delta^{\mathrm{M}} \boldsymbol{F} \cdot \overline{\boldsymbol{X}}\right] \cdot \overline{\boldsymbol{t}}_{0} \mathrm{~d} L \\
=\frac{1}{\mathscr{V}_{0}}\left[\int_{\mathcal{S}_{0}} \delta \boldsymbol{\varphi} \cdot \boldsymbol{t}_{0} \mathrm{~d} A-\delta^{\mathrm{M}} \boldsymbol{F}: \int_{\mathcal{S}_{0}} \boldsymbol{t}_{0} \otimes \boldsymbol{X} \mathrm{d} A\right. \\
\left.+\int_{\mathcal{L}_{0}} \delta \overline{\boldsymbol{\varphi}} \cdot \overline{\boldsymbol{t}}_{0} \mathrm{~d} L-\delta^{\mathrm{M}} \boldsymbol{F}: \int_{\mathcal{L}_{0}} \overline{\boldsymbol{t}}_{0} \otimes \overline{\boldsymbol{X}} \mathrm{d} L\right]
\end{aligned}
$$




$$
\begin{aligned}
& =\frac{1}{\mathscr{V}_{0}}[\int_{\mathcal{S}_{0}} \delta \boldsymbol{\varphi} \cdot \boldsymbol{t}_{0} \mathrm{~d} A-\delta^{\mathrm{M}} \boldsymbol{F}: \underbrace{\left[\int_{\mathcal{S}_{0}} \boldsymbol{t}_{0} \otimes \boldsymbol{X} \mathrm{d} A+\int_{\mathcal{L}_{0}} \overline{\boldsymbol{t}}_{0} \otimes \overline{\boldsymbol{X}} \mathrm{d} L\right]}_{\mathscr{V}_{0}{ }^{\mathrm{M}} \boldsymbol{P} \text { according to Equation }(4)_{2}}+\int_{\mathcal{L}_{0}} \delta \overline{\boldsymbol{\varphi}} \cdot \overline{\boldsymbol{t}}_{0} \mathrm{~d} L] \\
& =\frac{1}{\mathscr{V}_{0}}\left[\int_{\mathcal{S}_{0}} \delta \boldsymbol{\varphi} \cdot \boldsymbol{t}_{0} \mathrm{~d} A+\int_{\mathcal{L}_{0}} \delta \overline{\boldsymbol{\varphi}} \cdot \overline{\boldsymbol{t}}_{0} \mathrm{~d} L-\mathscr{V}_{0} \delta^{\mathrm{M}} \boldsymbol{F}:{ }^{\mathrm{M}} \boldsymbol{P}\right] \quad \text { using Lemma } 6 \\
& =\frac{1}{\mathscr{V}_{0}} \int_{\mathcal{B}_{0}} \boldsymbol{P}: \delta \boldsymbol{F} \mathrm{d} V+\frac{1}{\mathscr{V}_{0}} \int_{\mathcal{I}_{0}} \overline{\boldsymbol{P}}: \delta \overline{\boldsymbol{F}} \mathrm{d} A-{ }^{\mathrm{M}} \boldsymbol{P}: \delta^{\mathrm{M}} \boldsymbol{F} .
\end{aligned}
$$

\section{Appendix 3. Macro Piola tangent}

Performing a full $\mathrm{FE}^{2}$ simulation requires the solutions of both the macro- and the microproblem simultaneously. In order to solve the macro-problem, in addition to the macro Piola stress, the macro Piola tangent is required. To calculate the macro Piola tangent, we establish the average Piola tangent theorem as follows.

Theorem: Let ${ }^{M} \boldsymbol{F}$ and ${ }^{M} \boldsymbol{P}$ be the given macro deformation gradient tensor and macro Piola stress tensor, respectively. The micro deformation gradient may not be uniform within the bulk and on the interface within the RVE and can be decomposed into a uniform part and a zeromean fluctuation part as $\boldsymbol{F}={ }^{M} \boldsymbol{F}+\widetilde{\boldsymbol{F}}$. Let the fourth-order tensor $\mathbb{B}$ denote the linear map from $\delta^{M} \boldsymbol{F}$ to its fluctuation $\delta \widetilde{\boldsymbol{F}}$ as $\delta \widetilde{\boldsymbol{F}}=\mathbb{B}: \delta^{M} \boldsymbol{F}$. Accordingly, the macro Piola tangent ${ }^{M} \mathbb{A}$ is computed as

$$
{ }^{M} \mathbb{A}=\frac{1}{\mathscr{V}_{0}} \int_{\mathcal{B}_{0}} \mathbb{A}+\mathbb{A}: \mathbb{B} d V+\frac{1}{\mathscr{V}_{0}} \int_{\mathcal{I}_{0}} \overline{\mathbb{A}}+\overline{\mathbb{A}}: \mathbb{B} d A
$$

Proof: In order to prove the average Piola tangent theorem stated above, recall that $\delta^{\mathrm{M}} \boldsymbol{P}=$ ${ }^{\mathrm{M}} \mathbb{A}: \delta^{\mathrm{M}} \boldsymbol{F}$. Then, from the assumption that the variation of the macro Piola stress is the average of the variation of the micro Piola stress over the bulk and the interface of the RVE, we have

$$
\begin{aligned}
\delta^{\mathrm{M}} \boldsymbol{P} & =\frac{1}{\mathscr{V}_{0}} \int_{\mathcal{B}_{0}} \delta \boldsymbol{P} \mathrm{d} V+\frac{1}{\mathscr{V}_{0}} \int_{\mathcal{I}_{0}} \delta \overline{\boldsymbol{P}} \mathrm{d} A \\
& =\frac{1}{\mathscr{V}_{0}} \int_{\mathcal{B}_{0}} \mathbb{A}: \delta \boldsymbol{F} \mathrm{d} V+\frac{1}{\mathscr{V}_{0}} \int_{\mathcal{I}_{0}} \overline{\mathbb{A}}: \delta \overline{\boldsymbol{F}} \mathrm{d} A \\
& =\frac{1}{\mathscr{V}_{0}} \int_{\mathcal{B}_{0}} \mathbb{A}: \delta \boldsymbol{F} \mathrm{d} V+\frac{1}{\mathscr{V}_{0}} \int_{\mathcal{I}_{0}} \overline{\mathbb{A}}: \delta \boldsymbol{F} \cdot \overline{\boldsymbol{I}} \mathrm{d} A \\
& =\frac{1}{\mathscr{V}_{0}} \int_{\mathcal{B}_{0}} \mathbb{A}: \delta \boldsymbol{F} \mathrm{d} V+\frac{1}{\mathscr{V}_{0}} \int_{\mathcal{I}_{0}} \overline{\mathbb{A}}: \delta \boldsymbol{F} \mathrm{d} A \\
& =\frac{1}{\mathscr{V}_{0}} \int_{\mathcal{B}_{0}} \mathbb{A}:\left[\delta^{\mathrm{M}} \boldsymbol{F}+\delta \widetilde{\boldsymbol{F}}\right] \mathrm{d} V+\frac{1}{\mathscr{V}_{0}} \int_{\mathcal{I}_{0}} \overline{\mathbb{A}}:\left[\delta^{\mathrm{M}} \boldsymbol{F}+\delta \widetilde{\boldsymbol{F}}\right] \mathrm{d} A \\
& =\frac{1}{\mathscr{V}_{0}} \int_{\mathcal{B}_{0}} \mathbb{A}:\left[\delta^{\mathrm{M}} \boldsymbol{F}+\mathbb{B}: \delta^{\mathrm{M}} \boldsymbol{F}\right] \mathrm{d} V+\frac{1}{\mathscr{V}_{0}} \int_{\mathcal{I}_{0}} \overline{\mathbb{A}}:\left[\delta^{\mathrm{M}} \boldsymbol{F}+\mathbb{B}: \delta^{\mathrm{M}} \boldsymbol{F}\right] \mathrm{d} A \\
& =\underbrace{\left[\frac{1}{\mathscr{V}_{0}} \int_{\mathcal{B}_{0}} \mathbb{A}+\mathbb{A}: \mathbb{B} \mathrm{d} V+\frac{1}{\mathscr{V}_{0}} \int_{\mathcal{I}_{0}} \overline{\mathbb{A}}+\overline{\mathbb{A}}: \mathbb{B} \mathrm{d} A\right]: \delta^{\mathrm{M}} \boldsymbol{F} .}_{\mathrm{M}_{\mathbb{A}}}
\end{aligned}
$$


We determine $\mathbb{B}$ at the converged solution of the micro-problem and based on the linear relationship between $\delta^{\mathrm{M}} \boldsymbol{F}$ and $\delta \widetilde{\boldsymbol{F}}$. Thereby $\mathbb{B}$ is evaluated numerically by perturbing ${ }^{\mathrm{M}} \boldsymbol{F}$ and calculating the resultant $\delta \widetilde{\boldsymbol{F}}$ through solving a system of linear problems. 